\title{
Universality of Random Matrices and Local Relaxation Flow
}

\author{
László Erdős ${ }^{1 *}$ Benjamin Schlein ${ }^{2}$ and Horng-Tzer Yau ${ }^{3 \dagger}$ \\ Institute of Mathematics, University of Munich, \\ Theresienstr. 39, D-80333 Munich, Germany ${ }^{1}$ \\ Department of Pure Mathematics and Mathematical Statistics \\ University of Cambridge \\ Wilberforce Rd, Cambridge CB3 0WB, UK ${ }^{2}$ \\ Department of Mathematics, Harvard University \\ Cambridge MA 02138, USA $^{3}$
}

Nov 24, 2010

\begin{abstract}
Consider the Dyson Brownian motion with parameter $\beta$, where $\beta=1,2,4$ corresponds to the eigenvalue flows for the eigenvalues of symmetric, hermitian and quaternion self-dual ensembles. For any $\beta \geq 1$, we prove that the relaxation time to local equilibrium for the Dyson Brownian motion is bounded above by $N^{-\zeta}$ for some $\zeta>0$. The proof is based on an estimate of the entropy flow of the Dyson Brownian motion w.r.t. a "pseudo equilibrium measure". As an application of this estimate, we prove that the eigenvalue spacing statistics in the bulk of the spectrum for $N \times N$ symmetric Wigner ensemble is the same as that of the Gaussian Orthogonal Ensemble (GOE) in the limit $N \rightarrow \infty$. The assumptions on the probability distribution of the matrix elements of the Wigner ensemble are a subexponential decay and some minor restriction on the support.
\end{abstract}

AMS Subject Classification: 15A52, 82B44

Running title: Universality for Wigner matrices

Key words: Wigner random matrix, Dyson Brownian Motion.

\footnotetext{
*Partially supported by SFB-TR 12 Grant of the German Research Council

${ }^{\dagger}$ Partially supported by NSF grants DMS-0757425, 0804279
} 


\section{Introduction}

A central question concerning random matrices is the universality conjecture which states that local statistics of eigenvalues are determined by the symmetries of the ensembles but are otherwise independent of the details of the distributions. There are two types of universalities: the edge universality and the bulk universality concerning the interior of the spectrum. The edge universality is commonly approached via the moment method $[26,27]$ while the bulk universality was proven for very general classes of unitary invariant ensembles (see, e.g. [3, 6, 7, 24, 25] and references therein) based on detailed analysis of orthogonal polynomials. The most prominent non-unitary ensembles are the Wigner matrices, i.e., random matrices with i.i.d. matrix elements that follow a general distribution. The bulk universality for Hermitian Wigner ensembles was first established in [12] for ensembles with smooth distributions. The later work [28] by Tao and Vu did not assume smoothness but it required some moment condition which was removed later in [13]. Our approach [12] to prove the universality was based on the following three steps.

Step 1: Local semicircle law. It states that the number of eigenvalues in a spectral window containing about $N^{\varepsilon}$ eigenvalues is given by the semicircle law with a very high probability $[9,10]$. The factor $N^{\varepsilon}$ can be improved to any sufficiently large constant at the expense of deterioriation of the probability estimate.

Step 2: Universality for Gaussian divisible ensembles. The Gaussian divisible ensembles are given by matrices of the form

$$
\widehat{H}+\sqrt{s} V
$$

where $\widehat{H}$ is a Wigner matrix, $V$ is an independent standard GUE matrix and $s>0$. Johansson [21] and the later improvement in [2] proved that the bulk universality holds for ensembles of the form (1.1) if $s>0$ is independent of $N$. In the work [12], this result was extended to $s=N^{-1+\varepsilon}$ for any $\varepsilon>0$. The key ingredient for this extension was the local semicircle law.

Step 3: Approximation by Gaussian divisible ensembles. For any given Wigner matrix $H$, we find another Wigner matrix $\widehat{H}$ so that the eigenvalue statistics of $H$ and $\widehat{H}+\sqrt{s} V$ are close to each other. The choice of $\widehat{H}$ is given by a reverse heat flow argument.

Johansson's proof of the universality of Hermitian Wigner ensembles relied on the asymptotic analysis of an explicit formula by Brézin-Hikami $[5,21]$ for the correlation functions of the eigenvalues of $\widehat{H}+\sqrt{s} V$. Unfortunately, the similar formula for GOE is not very explicit and the corresponding result is not available. On the other hand, the eigenvalue distribution of the matrix $\widehat{H}+\sqrt{s} V$ is the same as that of $\widehat{H}+V(s)$, where the matrix elements of $V(s)$ are independent standard Brownian motions with variance $s / N$. Dyson observed that the evolution of the eigenvalues of the flow $s \rightarrow \widehat{H}+V(s)$ is given by a system of coupled stochastic differential equations (SDE), commonly called the Dyson Brownian motion (DBM) [8].

If we replace the Brownian motions by the Ornstein-Uhlenbeck processes, the resulting dynamics on the eigenvalues, which we still call DBM, has the GUE or GOE eigenvalue distributions as the invariant measures depending on the symmetry type of the ensembles. Thus the result of Johansson can be interpreted as stating that the local statistics of GUE is reached via DBM for time of order one. In fact, by analyzing the dynamics of DBM with ideas from the hydrodynamical limit, we have extended Johansson's result to $s \gg N^{-3 / 4}$ [11]. The key observation of [11] is that the local statistics of eigenvalues depend exclusively on the approach to local equilibrium. This method avoids the usage of explicit formulae for correlation functions, but the identification of local equilibria, unfortunately, still uses explicit representations of correlation functions by orthogonal polynomials (following e.g. [25]), and the extension to other ensembles is not a simple task. 
Therefore, the universality for symmetric random matrices remained open and the only partial result is Theorem 23 of [28] for Wigner matrices with the first four moments of the matrix elements matching those of GOE. The approach of [28] consisted of three similar steps as outlined above. For Step 2, it used the result of [21]. For Step 3, a four moment comparison theorem for individual eigenvalues was proved in [28] and the local semicircle law (Step 1) was one of the key inputs in this proof.

In this paper, we introduce a general approach to prove local ergodicity of DBM, partially motivated by the previous work [11]. In this approach the analysis of orthogonal polynomials or explicit formulae are completely eliminated and the method applies to both Hermitian and symmetric ensembles. In fact, the heart of the proof is a convex analysis and it applies to $\beta$-ensembles for any $\beta \geq 1$. The model specific information required to complete this approach involves only rough estimates on the accuracy of the local density of eigenvalues. We expect this method to apply to a very general class of models. More detailed explanations will be given in Section 3.

\section{Statement of Main Results}

To fix the notation, we will present the case of symmetric Wigner matrices; the modification to the Hermitian case is straightforward and will be omitted. The extension to the quaternion self-dual case is also standard, see, e.g. [14] for the notations and setup. On the other hand, the main theorem on DBM (Theorem 2.1) is valid for general $\beta$-ensembles. Thus all notations for matrices will be restricted to symmetric matrices but all results for flows will be stated and proved for general $\beta$-ensembles. We first explain our general result about DBM and in Section 2.2 we will present its application to Wigner matrices.

\subsection{Local ergodicity of Dyson Brownian motion}

The joint distributions of the eigenvalues $\mathbf{x}=\left(x_{1}, x_{2}, \ldots, x_{N}\right)$ of the Gaussian Unitary Ensemble (GUE) and the Gaussian Orthogonal Ensemble (GOE) are given by the following measure

$$
\mu=\mu_{N}^{(\beta)}(\mathrm{d} \mathbf{x})=\frac{e^{-N \mathcal{H}(\mathbf{x})}}{Z_{\beta}} \mathrm{d} \mathbf{x}, \quad \mathcal{H}(\mathbf{x})=\left[\beta \sum_{i=1}^{N} \frac{x_{i}^{2}}{4}-\frac{\beta}{N} \sum_{i<j} \log \left|x_{j}-x_{i}\right|\right],
$$

where $\beta=1$ for GOE and $\beta=2$ for GUE. We will sometimes use $\mu$ to denote the density of the measure as well, i.e., $\mu(\mathbf{x}) \mathrm{d} \mathbf{x}=\mu(\mathrm{d} \mathbf{x})$. We consider $\mu$ defined on the ordered set

$$
\Sigma_{N}:=\left\{\mathbf{x} \in \mathbb{R}^{N}: x_{1}<x_{2}<\ldots<x_{N}\right\},
$$

and this measure is well-defined for all $\beta>0$. The Dyson Brownian motion (DBM) is characterized by the generator

$$
L=\sum_{i=1}^{N} \frac{1}{2 N} \partial_{i}^{2}+\sum_{i=1}^{N}\left(-\frac{\beta}{4} x_{i}+\frac{\beta}{2 N} \sum_{j \neq i} \frac{1}{x_{i}-x_{j}}\right) \partial_{i}
$$

acting on $L^{2}(\mu)$. The DBM is reversible with respect to $\mu$ with the Dirichlet form

$$
D(f)=-\int f L f \mathrm{~d} \mu=\sum_{j=1}^{N} \frac{1}{2 N} \int\left(\partial_{j} f\right)^{2} \mathrm{~d} \mu
$$


where $\partial_{j}=\partial_{x_{j}}$. Notice that we have added a drift $\frac{\beta}{4} x_{i} \partial_{i}$ so that the DBM is reversible w.r.t. $\mu$. The original definition by Dyson in [8] was slightly different; it contained no drift term.

Denote the distribution of the process at the time $t$ by $f_{t}(\mathbf{x}) \mu(\mathrm{d} \mathbf{x})$. Then $f_{t}$ satisfies

$$
\partial_{t} f_{t}=L f_{t} .
$$

The corresponding stochastic differential equation for $\mathbf{x}(t)$ is now given by (see, e.g. Section 12.1 of [18])

$$
\mathrm{d} x_{i}=\frac{\mathrm{d} B_{i}}{\sqrt{N}}+\left[-\frac{\beta}{4} x_{i}+\frac{\beta}{2 N} \sum_{j \neq i} \frac{1}{x_{i}-x_{j}}\right] \mathrm{d} t, \quad 1 \leq i \leq N,
$$

where $\left\{B_{i}: 1 \leq i \leq N\right\}$ is a collection of independent Brownian motions. The well-posedness of DBM on $\Sigma_{N}$ has been proved in Section 4.3.1 of [18], see the Appendix for some more details. This step requires $\beta \geq 1$ which we will assume from now on.

The dynamics given by (2.4) and (2.5) with $\beta=1,2,4$ can be realized by the evolution of the eigenvalues of symmetric, hermitian and quaternion self-dual matrix ensembles, but the dynamics is well-defined for $\beta \geq 1$ independently of the original matrix models. Our main result, Theorem 2.1 , is valid for all $\beta \geq 1$.

The expectation with respect to the density $f_{t}$ will be denoted by $\mathbb{E}_{t}$ with $\mathbb{E}:=\mathbb{E}_{0}$. For any $k \geq 1$ we define the $k$-point correlation functions (marginals) of the probability measure $f_{t} \mathrm{~d} \mu$ by

$$
p_{t, N}^{(k)}\left(x_{1}, x_{2}, \ldots, x_{k}\right):=\int_{\mathbb{R}^{N-k}} f_{t}(\mathbf{x}) \mu(\mathbf{x}) \mathrm{d} x_{k+1} \ldots \mathrm{d} x_{N} .
$$

Similarly, the correlation functions of the equilibrium measure are denoted by

$$
p_{\mu, N}^{(k)}\left(x_{1}, x_{2}, \ldots, x_{k}\right):=\int_{\mathbb{R}^{N-k}} \mu(\mathbf{x}) \mathrm{d} x_{k+1} \ldots \mathrm{d} x_{N} .
$$

For the purpose of these definitions only, we have extended the measures $f_{t}(\mathbf{x}) \mu(\mathbf{x}) \mathrm{d} \mathbf{x}$ and $\mu(\mathbf{x}) \mathrm{d} \mathbf{x}$ from the ordered set $\Sigma_{N}$ to $\mathbb{R}^{N}$ by requiring that the resulting measure on $\mathbb{R}^{N}$ is symmetric w.r.t. all permutations of $\{1, \ldots N\}$ and with a slight abuse of notations, we use the same notation $f_{t}(\mathbf{x}) \mu(\mathbf{x})$ to denote the density of the symmetrized measure. Apart from this definition, we follow the convention that the measures are defined on $\Sigma_{N}$ and all integrations in this paper are on the set $\Sigma_{N}$ unless otherwise specified.

Recall that

$$
\varrho_{s c}(x):=\frac{1}{2 \pi} \sqrt{\left(4-x^{2}\right)_{+}}
$$

is the density of the semicircle law and it is well-known that $\varrho_{s c}$ is also the density w.r.t. the measure $\mu$ in the limit $N \rightarrow \infty$ for $\beta \geq 1$. Define

$$
n_{s c}(E):=\int_{-\infty}^{E} \varrho_{s c}(x) \mathrm{d} x,
$$

and let $\gamma_{j}$ be the classical location of the $j$-th eigenvalue

$$
\gamma_{j}=n_{s c}^{-1}(j / N) .
$$

Introduce the quantity

$$
Q:=\sup _{t \geq 0} N \int\left[\frac{1}{N} \sum_{j=1}^{N}\left|x_{j}-\gamma_{j}\right|\right]^{2} f_{t}(\mathbf{x}) \mathrm{d} \mu(\mathbf{x}) .
$$

Our key result on the local ergodicity of DBM is the following theorem. 
Theorem 2.1 (Local Ergodicity of Dyson Brownian Motion) Suppose the initial density $f_{0}$ satisfies $S_{\mu}\left(f_{0}\right):=\int f_{0} \log f_{0} \mathrm{~d} \mu \leq C N^{m}$ with some fixed exponent $m$ independent of $N$. Let $f_{t}$ be the solution of the forward equation (2.4). Suppose that the following three assumptions are satisfied for all sufficiently large $N$.

Assumption 1. For some $\mathfrak{a}>0$ we have

$$
Q \leq N^{-2 \mathfrak{a}} .
$$

Assumption 2. There exist constants $\mathfrak{b}>0$ and $\mathfrak{c}>0$ such that

$$
\sup _{t \geq 0} \int \mathfrak{1}\left\{\max _{j=1, \ldots N}\left|x_{j}-\gamma_{j}\right| \geq N^{-\mathfrak{b}}\right\} f_{t} \mathrm{~d} \mu \leq \exp \left[-N^{\mathfrak{c}}\right] .
$$

Assumption 3. For any compact subinterval $I_{0} \subset(-2,2)$, and for any $\delta>0, \sigma>0$ and $n \in \mathbb{N}$ there is a constant $C_{n}$ depending on $I_{0}, \delta$ and $\sigma$ such that for any interval $I \subset I_{0}$ with $|I| \geq N^{-1+\sigma}$ and for any $K \geq 1$, we have

$$
\sup _{t \geq 0} \int 1\left\{\mathcal{N}_{I} \geq K N|I|\right\} f_{t} \mathrm{~d} \mu \leq C_{n} K^{-n} .
$$

Let $E \in \mathbb{R}$ such that $|E|<2$ and let $0<b<2-|E|$. There exists a constant $\zeta>0$, depending only on $\mathfrak{a}$ and $\mathfrak{b}$, such that, for any integer $k \geq 1$ and for any compactly supported continuous test function $O: \mathbb{R}^{k} \rightarrow \mathbb{R}$ we have

$$
\begin{aligned}
\lim _{N \rightarrow \infty} \int_{E-b}^{E+b} \frac{\mathrm{d} E^{\prime}}{2 b} \int_{\mathbb{R}^{k}} \mathrm{~d} \alpha_{1} \ldots \mathrm{d} \alpha_{k} O\left(\alpha_{1}, \ldots, \alpha_{k}\right) \\
\quad \times \frac{1}{\varrho_{s c}(E)^{k}}\left(p_{t, N}^{(k)}-p_{\mu, N}^{(k)}\right)\left(E^{\prime}+\frac{\alpha_{1}}{N \varrho_{s c}(E)}, \ldots, E^{\prime}+\frac{\alpha_{k}}{N \varrho_{s c}(E)}\right)=0
\end{aligned}
$$

for $t=N^{-\zeta}$.

Convention. We will use the letters $C$ and $c$ to denote general positive constants whose precise values are irrelevant and they may change from line to line.

The relaxation time to global equilibrium for the DBM is order one in our scaling. The simplest way to see this is via the Bakry-Emery theorem [1] which states that, roughly speaking, the relaxation time is the inverse of the lower bound to the Hessian of the Hamiltonian $\mathcal{H}$. In our case $\mathcal{H}^{\prime \prime} \geq I$, and this implies that the relaxation time is order one. On the other hand, it was conjectured by Dyson [8] that the relaxation time to local equilibrium is of order $N^{-1}$. Theorem 2.1 asserts that the relaxation time to local equilibrium is less than $N^{-\zeta}$. Although this is far from proving Dyson's conjecture, it is the first effective estimate that shows that the local equilibrium is approached much faster than the global one. Moreover, this result suffices to prove the bulk universality of Wigner matrices when combining with the reverse heat flow ideas introduced in [12]. We remark that the concept of local equilibrium is used vaguely here and in Dyson's paper. In principle, there are many local equilibria depending on boundary conditions and the uniqueness is a tough question especially now that the interaction is long ranged and singular.

The proof of Theorem 2.1 is based on the introduction of the pseudo equilibrium measure which we now explain. It is common that the global and the local equilibrium are reached at different time scales 
for interacting particle systems, of which DBM is a special case. On the other hand, the hydrodynamical approach [11] for the DBM yields very complicated estimates. The main reason for the complications is due to that the equilibrium measure of DBM has a logarithmic two body interaction that is both long range and singular at short distances. Hence the proof of the uniqueness of "local equilibrium measures" is very complicated and we were able to carry it out only for the Hermitian case due to that several identities involving orthogonal polynomials are valid only for the $\beta=2$ case. However, there are two key observations from this study:

1. The local statistics does not depend on the long range part of the logarithmic interaction, in other words, we can cutoff the interactions between far away particles without changing the local statistics.

2. The relaxation time for the gradient flow associated with the local equilibrium with a fixed boundary condition is much smaller than the global relaxation time of the DBM, which is of order one.

To finesse the difficulty associated with the uniqueness of local equilibria, we define the pseudo equilibrium measure, $\omega$, by cutting off the long range interactions of the equilibrium measure $\mu$ and show that $\omega, \mu$ and $f_{t} \mu$ all have the same local statistics. The key idea that the last assertion holds is to estimate the relative entropy of the solution to the DBM, $f_{t} \mu$, relative to the pseudo equilibrium measure $\omega$. Since the pseudo equilibrium measure is not a global equilibrium measure, the entropy will not decay monotonically as in the case of the relative entropy w.r.t. the equilibrium measure. More precisely, the time derivative of the relative entropy, under the flow of DBM, w.r.t. the pseudo equilibrium measure consists of two terms (see Theorem 3.5): (i) a dissipation term of Dirichlet form of $f_{t} \mu$ w.r.t. the pseudo equilibrium measure; (ii) an error term due to the fact that the pseudo equilibrium measure is not the true equilibrium measure.

Since the logarithmic interactions between far away particles can be approximated by a mean-field potential obtained from using the local density, the error term in (ii) can be controlled if we know the local density of particles w.r.t the distribution $f_{t} \mu$. The precise conditions are the Assumptions (1)-(3). In the special case of $\beta=1,2$, when the DBM is generated by symmetric matrix ensembles, these assumptions will be verified in Lemma 2.2 by using the local semicircle law; the case of $\beta=4$ is similar and some details are given in [14]. For other values of $\beta$ it is an open question to verify the corresponding assumptions. Given that the error term in (ii) can be bounded, we obtain an estimate on the Dirichlet form of $f_{t} \mu$ w.r.t. the pseudo equilibrium measure. The key question is whether this estimate alone is sufficient to pin down the local statistics. For this purpose, we note that the Dirichlet form w.r.t. $\omega$ generates a new gradient flow, the local relaxation flow. The global relaxation time of the local relaxation flow, determined by the convexity of the pseudo equilibrium measure, is much shorter than that of the standard DBM. This leads to strong estimates on the local relaxation flow and in particular, it identifies the local statistics. The details of the entropy estimates and the local relaxation flow will appear in Section 3. We now state the main application of Theorem 2.1, the universality of symmetric Wigner ensembles.

\subsection{Universality of symmetric Wigner ensemble}

Fix $N \in \mathbb{N}$ and we consider the symmetric matrix ensemble of $N \times N$ matrices $H=\left(h_{\ell k}\right)$ with normalization given by

$$
h_{\ell k}=N^{-1 / 2} x_{\ell k},
$$

where $x_{\ell k}$ for $\ell<k$ are independent, identically distributed random variables with the distribution $\nu$ that has zero expectation and variance 1 . The diagonal elements $x_{\ell \ell}$ are also i.i.d. with distribution $\widetilde{\nu}$ that has 
zero expectation and variance two. We will assume that $\nu$ satisfy the logarithmic Sobolev inequality (LSI), i.e. that there is a constant $\theta$ such that for any nonnegative function $u$ with $\int u \mathrm{~d} \nu=1$ we have

$$
\int u \log u \mathrm{~d} \nu \leq \theta \int|\nabla \sqrt{u}|^{2} \mathrm{~d} \nu
$$

We remark that (2.15) implies [22] that $\nu$ has a Gaussian decay, i.e.

$$
D:=\int e^{\delta_{0} x^{2}} \mathrm{~d} \nu(x)<\infty
$$

for some $\delta_{0}>0$. We require that $\widetilde{\nu}$ also satisfies (2.15). In this paper, all conditions and statements involving $\nu$ apply to $\widetilde{\nu}$ as well, but for the simplicity of the presentation, we will neglect mentioning $\widetilde{\nu}$ all the times.

Suppose that the matrix element $h_{\ell k}$ of the symmetric ensembles evolves according to the OrnsteinUhlenbeck process on $\mathbb{R}$, i.e. the distribution $\nu_{t}=u_{t} \gamma$ at the time $t$ satisfies

$$
\partial_{t} u_{t}=A u_{t}, \quad A=\frac{1}{2} \frac{\partial^{2}}{\partial x^{2}}-\frac{x}{2} \frac{\partial}{\partial x}
$$

where $\gamma$ is the standard Gaussian distribution with variance one. For the diagonal element, the OrnsteinUhlenbeck process should be replaced by the one reversible w.r.t. the Gaussian measure with variance two due to the convention that the variances of the diagonal elements are equal to two. The Ornstein-Uhlenbeck process (2.17) induces a stochastic process on the eigenvalues; it is well-known that the process on the eigenvalue is given by the $\operatorname{DBM}(2.5)$ with $\beta=1$. Notice that we used the Ornstein-Uhlenbeck process so that the resulting DBM is reversible w.r.t. $\mu$.

Our goal is to apply Theorem 2.1 with $\beta=1$ and for this purpose, we need to verify Assumptions 1-3. Assumption 3 follows from the local semicircle law, Theorem 5.1, stated later in Section 5. Assumptions 1 and 2 can be verified if the measure $\nu$ satisfies the logarithmic Sobolev inequality (2.15). The precise statement is the following lemma.

Lemma 2.2 Suppose the assumption (2.15) on the distribution $\nu$ of the matrix elements holds. Then there are positive numbers $\mathfrak{a}, \mathfrak{b}$ and $\mathfrak{c}$, depending on $\theta$ from (2.15), such that (2.10) and (2.11) hold.

From this Lemma, for symmetric Wigner matrices whose matrix element distributions satisfy the LSI, the assumptions of Theorem 2.1 are satisfied. Hence the correlation functions w.r.t. $f_{t} \mu$ and the GOE equilibrium measure $\mu_{N}^{(\beta=1)}$ are identical in the large $N$ limit for some $t=N^{-\zeta}$ in the sense that (2.13) holds. Together with the reverse heat flow argument, we have the following universality theorem for local statistics of Wigner ensembles whose matrix element distribution is smooth and satisfies the logarithmic Sobolev inequality. Denote by $p_{N}^{(k)}$ the correlation functions of the eigenvalues of the symmetric Wigner ensemble. Let $p_{N, G O E}^{(k)}$ be the correlation functions of the eigenvalues of GOE, i.e., the correlation functions of the equilibrium measure $\mu_{N}^{(\beta=1)}$. It is well-known that $p_{N, G O E}^{(k)}$ can be computed explicitly (see, e.g. Section 7 of [24]).

Theorem 2.3 Suppose the distribution $\nu$ for the matrix elements satisfies the logarithmic Sobolev inequality (2.15). Assume that $\nu$ has a positive density $\nu(x)=e^{-U(x)}$ such that for any $j$ there are constants $C_{1}, C_{2}$, depending on $j$, such that

$$
\left|U^{(j)}(x)\right| \leq C_{1}\left(1+x^{2}\right)^{C_{2}} .
$$


Then for any $|E|<2$ and for any $k \geq 1$, we have

$$
\begin{aligned}
\lim _{b \rightarrow 0} \lim _{N \rightarrow \infty} \frac{1}{2 b} \int_{E-b}^{E+b} & \int_{\mathbb{R}^{k}} O\left(\alpha_{1}, \ldots, \alpha_{k}\right) \\
& \times \frac{1}{\left[\varrho_{s c}\left(E^{\prime}\right)\right]^{k}}\left(p_{N}^{(k)}-p_{N, G O E}^{(k)}\right)\left(E^{\prime}+\frac{\alpha_{1}}{N \varrho_{s c}\left(E^{\prime}\right)}, \ldots, E^{\prime}+\frac{\alpha_{k}}{N \varrho_{s c}\left(E^{\prime}\right)}\right) \mathrm{d} \alpha_{1} \ldots \mathrm{d} \alpha_{k} \mathrm{~d} E^{\prime}=0,
\end{aligned}
$$

where $O: \mathbb{R}^{k} \rightarrow \mathbb{R}$ is an arbitrary continuous, compactly supported function.

Theorem 2.3 is a simple corollary of Theorem 2.1 and the method of the reverse heat flow [12]. It will be proved briefly in Section 4. Though we stated the universality in terms of correlation functions, it also holds for the eigenvalue gap distribution and we omit the obvious statement (the analogous statement for the Hermitian case was formulated in Theorem 1.2 of [12]).

In the following corollary, by using Theorem 15 of [28], we remove all assumptions from Theorem 2.3 except for a decay condition and a technical condition that $\nu$ is supported in at least three points. This latter technical condition was removed in our later paper [16], where we generalized our approach to a broader class of random matrix ensembles.

Corollary 2.4 Suppose the distribution $\nu$ of the matrix elements has mean zero, variance one and a tail with a subexponential decay, i.e. it satisfies that

$$
\int_{\mathbb{R}} \mathbf{1}(|x| \geq y) \mathrm{d} \nu(x) \leq C \exp \left[-y^{\mathfrak{q}}\right], \quad \forall y \geq 0
$$

for some constants $C, \mathfrak{q}>0$. Assume that $\nu$ is supported in at least three points. Then the conclusion (2.19) of Theorem 2.3 holds.

Proof. Let $m_{j}$ denote the moments of $\nu$

$$
m_{j}:=\int_{\mathbb{R}} x^{j} \mathrm{~d} \nu
$$

where $m_{1}=0$ and $m_{2}=1$. It is easy to check that $m_{4} \geq m_{3}^{2}+1$ with the equality holds only for Bernoulli type distribution supported in two points. Due to the condition that $\nu$ is supported on at least three points, we thus have $m_{4}>m_{3}^{2}+1$. For fixed numbers $m_{3}, m_{4}$ satisfying $m_{4}>m_{3}^{2}+1$, there exists a probability measure $\widehat{\nu}$ on $\mathbb{R}$ with density $e^{-U(x)}$ such that (i) the first four moments of $\widehat{\nu}$ match to those of $\nu$, i.e.

$$
\int_{\mathbb{R}} x \mathrm{~d} \widehat{\nu}=0, \quad \int_{\mathbb{R}} x^{2} \mathrm{~d} \widehat{\nu}=1, \quad \int_{\mathbb{R}} x^{j} \mathrm{~d} \widehat{\nu}=m_{j}, \quad j=3,4 ;
$$

(ii) the derivative bounds (2.18) hold, and (iii) the logarithmic Sobolev inequality (2.15) holds. It is easy to argue that such a measure $\widehat{\nu}$ exists. Consider the space of all measures satisfying (2.18) with a finite LSI constant. Since the condition (2.18) and the finite LSI constant condition are preserved under small smooth perturbations which are infinite dimensional, there are enough freedom to choose perturbations so as to match the first four moments as long as $m_{4}>m_{3}^{2}+1$. An elementary detailed proof of this fact is 
given in Lemma C.1. of [15]. Therefore, $\widehat{\nu}$ satisfies the assumption of Theorem 2.3 and thus (2.19) holds for the measure $\widehat{\nu}$. Recall that Theorem 15 in [28] asserts that the local eigenvalue statistics for matrices whose matrix element distributions match up to the first four moments are the same in the limit $N \rightarrow \infty$ (strictly speaking, this theorem was proved only for hermitian matrices, but the parallel version for symmetric ensembles holds as well, see the remark at the end of Section 1.6 in [28]). This proves the corollary.

\section{$3 \quad$ Pseudo equilibrium measure and Entropy Dissipation Estimates}

The key idea to prove Theorem 2.1 is an estimate on the time to local equilibrium for the DBM. However, to estimate this time to local equilibrium, we need to introduce a different flow, the local relaxation flow, defined as the gradient flow of the pseudo equilibrium measure. The pseudo equilibrium measure is a measure which has the local statistics of the $\beta$ ensemble but has a strong convexity property. Fix a positive number $\eta$ with $N^{-1 / 6} \ll \eta \ll 1$, and for the rest of this paper let $\varepsilon>0$ be a small positive number which we will not specify. Let $\gamma_{j}^{ \pm}:=\gamma_{j} \pm \eta N^{-\varepsilon}$ and define the mean field potential of eigenvalues far away from the $j$-th one as

$$
W_{j}(x):=-\frac{\beta}{N} \sum_{k:|k-j|>N \eta} \log \left(\left|x-\gamma_{k}\right|+\eta\right) \quad \text { if } \quad x \in I_{j}:=\left(\gamma_{j}^{-}, \gamma_{j}^{+}\right)
$$

where the summation is over all $k \in\{1,2, \ldots, N\}$ such that $|k-j|>N \eta$. For $x \geq \gamma_{j}^{+}$, we extend $W_{j}$ by

$$
W_{j}(x)=W_{j}\left(\gamma_{j}^{+}\right)+W_{j}^{\prime}\left(\gamma_{j}^{+}\right)\left(x-\gamma_{j}^{+}\right)+\frac{W^{\prime \prime}\left(\gamma_{j}^{+}\right)}{2}\left(x-\gamma_{j}^{+}\right)^{2}
$$

and similarly for $x \leq \gamma_{j}^{-}$. In other words, $W_{j}$ is just the simplest convex extension of the function defined by (3.1) on $I_{j}$. This modification will avoid the singularities at $x=\gamma_{k}$. Notice that this is purely a technical device since we will show in (5.26) of Proposition 5.9 that the probability of the regime $I_{j}^{c}$ is negligible in the sense that

$$
\sum_{j=1}^{N} \int \mathbf{1}\left(x_{j} \notin I_{j}\right) f_{t} \mathrm{~d} \mu \leq C e^{-c N^{\varepsilon}} .
$$

The pseudo equilibrium measure $\omega_{N}=\omega$ is defined by

$$
\omega=\frac{1}{\widetilde{Z}} \exp \left[-N \sum_{j=1}^{N}\left\{\beta \frac{x_{j}^{2}}{4}+W_{j}\left(x_{j}\right)\right\}+\beta \sum_{i<j} \log \left|x_{i}-x_{j}\right|-\frac{\beta}{2} \sum_{i} \sum_{j:|j-i|>N \eta} \log \left(\left|x_{i}-x_{j}\right|+\eta\right)\right]=: \frac{e^{-\widetilde{\mathcal{H}}}}{\widetilde{Z}}
$$

We can write $\omega=\psi \mu$ with

$$
\psi=\frac{Z_{\beta}}{\widetilde{Z}} \exp \left[-\frac{\beta}{2} \sum_{i=1}^{N} \sum_{j:|j-i|>N \eta} \log \left(\left|x_{i}-x_{j}\right|+\eta\right)-N \sum_{i=1}^{N} W_{i}\left(x_{i}\right)\right] .
$$

Recall that the relative entropy with respect to a measure $\lambda$ is defined by

$$
S_{\lambda}(f)=\int f \log f \mathrm{~d} \lambda, \quad S_{\lambda}(f \mid \psi)=\int f \log (f / \psi) \mathrm{d} \lambda
$$


and the Dirichlet form

$$
D_{\lambda}(h)=\frac{1}{2 N} \int(\nabla h)^{2} \mathrm{~d} \lambda=\frac{1}{2 N} \sum_{j=1}^{N} \int\left(\partial_{j} h\right)^{2} \mathrm{~d} \lambda .
$$

The local relaxation flow is defined to be the reversible dynamics w.r.t. $\omega$ characterized by the generator $\widetilde{L}$ defined by

$$
\int f \widetilde{L} g \mathrm{~d} \omega=-\frac{1}{2 N} \sum_{j=1}^{N} \int \partial_{j} f \partial_{j} g \mathrm{~d} \omega
$$

Explicitly, $\widetilde{L}$ is given by

$$
\widetilde{L}=L-\sum_{j=1}^{N} b_{j} \partial_{j}, \quad b_{j}:=\frac{\beta}{N} \sum_{k:|k-j|>N \eta} \frac{\operatorname{sgn}\left(x_{j}-x_{k}\right)}{\left|x_{j}-x_{k}\right|+\eta}+W_{j}^{\prime}\left(x_{j}\right),
$$

where

$$
W_{j}^{\prime}(x)=-\frac{\beta}{N} \sum_{k:|k-j|>N \eta} \frac{\operatorname{sgn}\left(x-\gamma_{k}\right)}{\left|x-\gamma_{k}\right|+\eta}
$$

for $x \in I_{j}$. Note that for any $k$ with $|k-j|>N \eta$, we have $\gamma_{k} \notin 2 I_{j}$, where $2 I_{j}$ is the doubling of the interval $I_{j}$. Moreover, for $k=j \pm N \eta$ we have $\left|\gamma_{k}-\gamma_{j}\right| \leq C \eta^{2 / 3}$ for some constant $C$, and so $\left|x-\gamma_{k}\right| \leq C \eta^{2 / 3}$ for $x \in I_{j}$. Thus we obtain that

$$
\inf _{x \in I_{j}} W_{j}^{\prime \prime}(x) \geq c \inf _{|x| \leq 2+\eta} \int_{|x-\gamma| \geq C \eta^{2 / 3}} \frac{\varrho_{s c}(\gamma) \mathrm{d} \gamma}{|x-\gamma|^{2}} \geq c \eta^{-1 / 3}
$$

with some positive constant $c$, using $\beta \geq 1$. Since $W_{j}$ was defined by a convex extension outside $I_{j}$, the same bound holds for any $x$ :

$$
\inf _{j} \inf _{x \in \mathbb{R}} W_{j}^{\prime \prime}(x) \geq c \eta^{-1 / 3}
$$

i.e., the mean field potential is uniformly convex with the convexity bound given in (3.8).

The potential $W$ is chosen to satisfy the two convexity properties: (3.8) and (3.18) and there are many other possible choices for $W$. For example, without changing the form of $W$ given in (3.1), a more natural choice for $\gamma_{j}$ would be

$$
\gamma_{j}=\int x_{j} f_{0} \mathrm{~d} \mu .
$$

This may somewhat improve the constant in the estimate (2.10), but the analysis is more complicated and we will not pursue this choice in this paper.

\subsection{Local Ergodicity of Dyson Brownian Motion}

The following theorem is our main result on the local ergodicity of DBM.

Theorem 3.1 Suppose that $S_{\mu}\left(f_{0} \mid \psi\right) \leq C N^{m}$ for some $m$ fixed. Let $\tau:=\eta^{1 / 3} N^{\delta}$ for some $\delta>0$. Define

$$
\Lambda:=\sup _{t \leq \tau} \sum_{j=1}^{N} \int b_{j}^{2} f_{t} \mathrm{~d} \mu .
$$


Fix $n \geq 1$, let $G: \mathbb{R}^{n} \rightarrow \mathbb{R}$ be a bounded smooth function with compact support and define

$$
\mathcal{G}_{i, n}(\mathbf{x}):=G\left(N\left(x_{i}-x_{i+1}\right), N\left(x_{i+1}-x_{i+2}\right), \ldots, N\left(x_{i+n-1}-x_{i+n}\right)\right) .
$$

Then for any $J \subset\{1,2, \ldots, N-n\}$ we have

$$
\left|\int \frac{1}{N} \sum_{i \in J} \mathcal{G}_{i, n}(\mathbf{x}) f_{\tau} \mathrm{d} \mu-\int \frac{1}{N} \sum_{i \in J} \mathcal{G}_{i, n}(\mathbf{x}) \mathrm{d} \mu\right| \leq C N^{\delta / 2} \Lambda^{1 / 2}+C e^{-c N^{\delta}}
$$

We emphasize that Theorem 3.1 applies to all $\beta \geq 1$ ensembles and the only assumption concerning the distribution $f_{t}$ is in (3.9). Notice that the first error term becomes large for $\delta$ large, i.e., if $\tau$ is large. The first ingredient to prove Theorem 3.1 is the analysis of the local relaxation flow. The following theorem shows that the local relaxation flow satisfies an entropy dissipation estimate and its equilibrium measure satisfies the logarithmic Sobolev inequality.

Theorem 3.2 (Dirichlet Form Dissipation Estimate) Suppose (3.8) holds. Consider the equation

$$
\partial_{t} q_{t}=\widetilde{L} q_{t}
$$

with reversible measure $\omega$. Denote by $R:=\eta^{1 / 6}$. Then we have

$$
\begin{gathered}
\partial_{t} D_{\omega}\left(\sqrt{q_{t}}\right) \leq-C R^{-2} D_{\omega}\left(\sqrt{q_{t}}\right)-\frac{1}{2 N^{2}} \int \sum_{|i-j| \leq N \eta} \frac{1}{\left(x_{i}-x_{j}\right)^{2}}\left(\partial_{t} \sqrt{q_{t}}-\partial_{j} \sqrt{q_{t}}\right)^{2} \mathrm{~d} \omega, \\
\frac{1}{2 N^{2}} \int_{0}^{\infty} \mathrm{d} s \int_{|i-j| \leq N \eta} \frac{1}{\left(x_{i}-x_{j}\right)^{2}}\left(\partial_{i} \sqrt{q_{s}}-\partial_{j} \sqrt{q_{s}}\right)^{2} \mathrm{~d} \omega \leq D_{\omega}\left(\sqrt{q_{0}}\right)
\end{gathered}
$$

and the logarithmic Sobolev inequality

$$
S_{\omega}(q) \leq C R^{2} D_{\omega}(\sqrt{q})
$$

with a universal constant $C$. Thus the relaxation time to equilibrium is of order $R^{2}=\eta^{1 / 3}$ and we have

$$
S_{\omega}\left(q_{t}\right) \leq e^{-C t R^{-2}} S_{\omega}\left(q_{0}\right) .
$$

The notation $R=\eta^{1 / 6}$ was introduced so that this result and Theorem 4.2 in [14] are identical. The scale parameter $R$ has a meaning in [14], but it is purely a choice of convention here. The proof given below follows the argument in [1] and it was outlined in this context in Section 5.1 of [11]. The new observation is the additional second term on the r.h.s of (3.13), corresponding to "local Dirichlet form dissipation". The estimate (3.14) on this additional term will play a key role in this paper.

Proof. In [11] it was shown that, with the notation $h=\sqrt{q}$, we have

$$
\partial_{t} h=\widetilde{L} h+\frac{1}{2 N} h^{-1}(\nabla h)^{2}
$$

and

$$
\partial_{t} \frac{1}{2 N} \int(\nabla h)^{2} e^{-\widetilde{\mathcal{H}}} \mathrm{d} \mathbf{x} \leq-\frac{1}{2 N^{2}} \int \nabla h\left(\nabla^{2} \widetilde{\mathcal{H}}\right) \nabla h e^{-\widetilde{\mathcal{H}}} \mathrm{d} \mathbf{x} .
$$


In our case, (3.8) and the fact that

$$
\frac{\mathrm{d}^{2}}{\mathrm{~d} x^{2}}(\log (|x|+\eta)-\log |x|) \geq 0, \quad x>0,
$$

imply that the Hessian of $\widetilde{\mathcal{H}}$ is bounded from below as

$$
\frac{1}{2 N^{2}} \nabla h\left(\nabla^{2} \widetilde{\mathcal{H}}\right) \nabla h \geq C \eta^{-1 / 3} \frac{1}{N} \sum_{j}\left(\partial_{j} h\right)^{2}+\frac{1}{2 N^{2}} \sum_{|i-j| \leq N \eta} \frac{1}{\left(x_{i}-x_{j}\right)^{2}}\left(\partial_{i} h-\partial_{j} h\right)^{2}
$$

with some positive constant $C$. This proves (3.13) and (3.14) since $R^{2}=\eta^{1 / 3}$. Inserting the inequality

$$
\partial_{t} D_{\omega}\left(\sqrt{q_{t}}\right) \leq-C R^{-2} D_{\omega}\left(\sqrt{q_{t}}\right)
$$

from (3.13) into the equation

$$
\partial_{t} S_{\omega}\left(q_{t}\right)=-4 D_{\omega}\left(\sqrt{q_{t}}\right),
$$

and integrating the resulting equation, we prove (3.15). Inserting (3.15) into (3.19) we have

$$
\partial_{t} S_{\omega}\left(q_{t}\right) \leq-C R^{-2} S_{\omega}\left(q_{t}\right)
$$

and we obtain (3.16).

Remark 3.3 The proof of (3.17) requires an integration by parts and the boundary term at $x_{i}=x_{j}$ (explained in Section 5.1. of [11]) should vanish. In the Appendix we will justify this technical step.

Lemma 3.4 Suppose that the density $q_{0}$ satisfies $S_{\omega}\left(q_{0}\right) \leq C N^{m}$ with some $m>0$ fixed. For a fixed $n \geq 1$ let $G: \mathbb{R}^{n} \rightarrow \mathbb{R}$ be a bounded smooth function with compact support and recall the definition of $\mathcal{G}_{i, n}$ from (3.10). Let $J \subset\{1,2, \ldots, N-n\}$ and set $\tau=\eta^{1 / 3} N^{\delta}$. Then we have

$$
\left|\int \frac{1}{N} \sum_{i \in J} \mathcal{G}_{i, n}(\mathbf{x}) \mathrm{d} \omega-\int \frac{1}{N} \sum_{i \in J} \mathcal{G}_{i, n}(\mathbf{x}) q_{0} \mathrm{~d} \omega\right| \leq C \sqrt{\frac{D_{\omega}\left(\sqrt{q_{0}}\right) \tau}{N}}+C e^{-c N^{\delta}}
$$

with some constant $C$ depending only on $G$.

Proof. Without loss of generality, we consider only the case $J=\{1, \ldots, N-n\}$. Let $q_{t}$ satisfy

$$
\partial_{t} q_{t}=\widetilde{L} q_{t}
$$

with an initial condition $q_{0}$. We first compare $q_{\tau}$ with $q_{\infty}=1$. Using the entropy inequality,

$$
\int|q-1| \mathrm{d} \omega \leq 2 \sqrt{S_{\omega}(q)}
$$

and the exponential decay of the entropy (3.16), we have

$$
\left|\int \frac{1}{N} \sum_{i \in J} \mathcal{G}_{i, n}(\mathbf{x}) q_{\tau} \mathrm{d} \omega-\int \frac{1}{N} \sum_{i \in J} \mathcal{G}_{i, n}(\mathbf{x}) \mathrm{d} \omega\right| \leq C\left(N^{m} e^{-\tau \eta^{-1 / 3}}\right)^{1 / 2} \leq C e^{-c N^{\varepsilon}} .
$$


To compare $q_{0}$ with $q_{\tau}$, by differentiation, we have

$$
\begin{aligned}
& \int \frac{1}{N} \sum_{i \in J} \mathcal{G}_{i, n}(\mathbf{x}) q_{\tau} \mathrm{d} \omega-\int \frac{1}{N} \sum_{i \in J} \mathcal{G}_{i, n}(\mathbf{x}) q_{0} \mathrm{~d} \omega \\
& =\int_{0}^{\tau} \mathrm{d} s \int \frac{1}{N} \sum_{i \in J} \sum_{k=1}^{n} \partial_{k} G\left(N\left(x_{i}-x_{i+1}\right), \ldots, N\left(x_{i+n-1}-x_{i+n}\right)\right)\left[\partial_{i+k-1} q_{s}-\partial_{i+k} q_{s}\right] \mathrm{d} \omega .
\end{aligned}
$$

From the Schwarz inequality and $\partial q=2 \sqrt{q} \partial \sqrt{q}$ the last term is bounded by

$$
\begin{aligned}
2 \sum_{k=1}^{n} & {\left[\int_{0}^{\tau} \mathrm{d} s \int \sum_{i \in J}\left[\partial_{k} G\left(N\left(x_{i}-x_{i+1}\right), \ldots, N\left(x_{i+n-1}-x_{i+n}\right)\right)\right]^{2}\left(x_{i+k-1}-x_{i+k}\right)^{2} q_{s} \mathrm{~d} \omega\right]^{1 / 2} } \\
& \times\left[\int_{0}^{\tau} \mathrm{d} s \int \frac{1}{N^{2}} \sum_{i \in J} \frac{1}{\left(x_{i+k-1}-x_{i+k}\right)^{2}}\left[\partial_{i+k-1} \sqrt{q_{s}}-\partial_{i+k} \sqrt{q_{s}}\right]^{2} \mathrm{~d} \omega\right]^{1 / 2} \\
\leq & C_{n} \sqrt{\frac{D_{\omega}\left(\sqrt{q_{0}}\right) \tau}{N}}
\end{aligned}
$$

where we have used (3.14) and that

$$
\left[\partial_{k} G\left(N\left(x_{i}-x_{i+1}\right), \ldots, N\left(x_{i+k-1}-x_{i+k}\right), \ldots N\left(x_{i+n-1}-x_{i+n}\right)\right)\right]^{2}\left(x_{i+k-1}-x_{i+k}\right)^{2} \leq C N^{-2}
$$

since $G$ is smooth and compactly supported. This proves the Lemma.

Notice if we use only the entropy dissipation and Dirichlet form, the main term on the right hand side of (3.20) will become $C \sqrt{S_{\omega}(q) \tau}$. Hence by exploiting the Dirichlet form dissipation coming from the second term on the r.h.s. of (3.13), we gain the crucial factor $N^{-1 / 2}$ in the estimate.

The second ingredient to prove Theorem 3.1 is the following entropy and Dirichlet form estimates.

Theorem 3.5 (Entropy and Dirichlet Form Estimates) Suppose the assumptions of Theorem 3.1 hold. Recall that $\tau=\eta^{1 / 3} N^{\delta}$ and define $g_{t}:=f_{t} / \psi$ so that $S_{\mu}\left(f_{t} \mid \psi\right)=S_{\omega}\left(g_{t}\right)$. Then the entropy and the Dirichlet form satisfy the estimates:

$$
\begin{gathered}
S_{\omega}\left(g_{\tau / 2}\right) \leq C N R^{2} \Lambda, \\
D_{\omega}\left(\sqrt{g_{\tau}}\right) \leq C N \Lambda .
\end{gathered}
$$

Proof. First we need the following relative entropy identity from [31].

Lemma 3.6 Let $f_{t}$ be a probability density satisfying $\partial_{t} f_{t}=L f_{t}$. Then for any probability density $\psi_{t}$ we have

$$
\partial_{t} S_{\mu}\left(f_{t} \mid \psi_{t}\right)=-\frac{2}{N} \sum_{j} \int\left(\partial_{j} \sqrt{g_{t}}\right)^{2} \psi_{t} \mathrm{~d} \mu+\int g_{t}\left(L-\partial_{t}\right) \psi_{t} \mathrm{~d} \mu
$$

where $g_{t}=f_{t} / \psi_{t}$. 
In our setting, $\psi$ is independent of $t$ and $L$ satisfies (3.6). Hence we have

$$
\partial_{t} S_{\omega}\left(g_{t}\right)=\partial_{t} S_{\mu}\left(f_{t} \mid \psi\right)=-\frac{2}{N} \sum_{j} \int\left(\partial_{j} \sqrt{g_{t}}\right)^{2} \mathrm{~d} \omega+\int \widetilde{L} g_{t} \mathrm{~d} \omega+\sum_{j} \int b_{j} \partial_{j} g_{t} \mathrm{~d} \omega .
$$

Since the middle term on the right hand side vanishes, we have from the Schwarz inequality

$$
\partial_{t} S_{\omega}\left(g_{t}\right) \leq-D_{\omega}\left(\sqrt{g_{t}}\right)+C N \sum_{j} \int b_{j}^{2} g_{t} \mathrm{~d} \omega .
$$

Together with the LSI (3.15) and (3.9), we have

$$
\partial_{t} S_{\omega}\left(g_{t}\right) \leq-C R^{-2} S_{\omega}\left(g_{t}\right)+C N \Lambda
$$

for $t \leq \tau$. Since $S_{\omega}\left(g_{0}\right)=S_{\mu}\left(f_{0} \mid \psi\right) \leq C N^{m}$ and $\tau / 2 \gg R^{2}$, the last inequality proves (3.22).

Integrating (3.25) from $t=\tau / 2$ to $t=\tau$ and using the monotonicity of the Dirichlet form in time, we have proved (3.23) with the choice of $\tau$.

Proof of Theorem 3.1. Fix $\tau=R^{2} N^{\delta}=\eta^{1 / 3} N^{\delta}$ and let $q_{0}:=g_{\tau}=f_{\tau} / \psi$ with $f_{0}$ satisfying the assumption of Theorem 3.5, i.e., $S_{\mu}\left(f_{0} \mid \psi\right) \leq C N^{m}$ for some $m$ and (3.9) holds. Using (3.23), we have

$$
\sqrt{\frac{D_{\omega}\left(\sqrt{q_{0}}\right) \tau}{N}} \leq C N^{\delta / 2} \Lambda^{1 / 2}
$$

and from (3.20) we also have

$$
\left|\int \frac{1}{N} \sum_{i \in J} \mathcal{G}_{i, n}(\mathbf{x}) f_{\tau} \mathrm{d} \mu-\int \frac{1}{N} \sum_{i \in J} \mathcal{G}_{i, n}(\mathbf{x}) \mathrm{d} \omega\right| \leq C N^{\delta / 2} \Lambda^{1 / 2}+C e^{-c N^{\delta}} .
$$

Clearly, equation (3.27) also holds for the special choice $f_{0}=1$ (for which $f_{\tau}=1$ ), i.e. local statistics of $\mu$ and $\omega$ can be compared. Hence we can replace the measure $\omega$ in (3.27) by $\mu$ and this proves Theorem 3.1.

\section{Proof of Theorem 2.3 and Theorem 2.1}

We first prove Theorem 2.3 assuming that Theorem 2.1 holds. Our main tool is the reverse heat flow argument from [12]. Recall that the distribution of the matrix element is given by a measure $\nu$ and the generator of the Ornstein-Uhlenbeck process is $A(2.17)$. The probability distribution of all matrix elements is $\nu^{\otimes n}, n=N^{2}$. The joint probability distribution of the matrix elements at time $t$ as every matrix element evolves under the Ornstein-Uhlenbeck process is given by

$$
F_{t} \mathrm{~d} \gamma^{\otimes n}:=\left(e^{t A} u\right)^{\otimes n} \mathrm{~d} \gamma^{\otimes n},
$$

where we recall that $\gamma$ is the standard Gaussian measure. 
Proposition 4.1 Fix a positive integer $K$. Suppose that $\nu=u \mathrm{~d} \gamma$ satisfies the subexponential decay condition (2.20) and the regularity condition (2.18) for all $j \leq K$. Then there is a small constant $\alpha_{K}$, depending only on $K$, such that for any positive $t \leq \alpha_{K}$ there exists a probability density $g_{t}$ w.r.t. $\gamma$ with mean zero and variance one such that

$$
\int\left|e^{t A} g_{t}-u\right| \mathrm{d} \gamma \leq C t^{K}
$$

for some $C>0$ depending on $K$. Furthermore, $g_{t}$ can be chosen such that if the logarithmic Sobolev inequality (2.15) holds for the measure $\nu=u \gamma$, then it holds for $g_{t} \gamma$ as well, with the logarithmic Sobolev constant changing by a factor of at most 2 .

Furthermore, let $\mathcal{A}=A^{\otimes n}, F=u^{\otimes n}$ with $n=N^{2}$ and set $G_{t}:=g_{t}^{\otimes n}$. Then we also have

$$
\int\left|e^{t \mathcal{A}} G_{t}-F\right| \mathrm{d} \gamma^{\otimes n} \leq C N^{2} t^{K}
$$

for some $C>0$ depending on $K$.

Proof. This proposition can be proved following the reverse heat flow idea from [12]. Define $\theta(x)=$ $\theta_{0}\left(t^{\alpha} x\right)$ with some small positive $\alpha>0$ depending on $K$, where $\theta_{0}$ is a smooth cutoff function satisfying $\theta_{0}(x)=1$ for $|x| \leq 1$ and $\theta_{0}(x)=0$ for $|x| \geq 2$. Set

$$
h_{s}=u+\theta \xi_{s}, \quad \text { with } \quad \xi_{s}:=\left[-s A+\frac{1}{2} s^{2} A^{2}+\ldots+(-1)^{K-1} \frac{s^{K-1}}{(K-1) !} A^{K-1}\right] u .
$$

By assumption (2.18), $h_{s}$ is positive and

$$
\frac{2}{3} u \leq h_{s} \leq \frac{3}{2} u
$$

for any $s \leq t$ if $t$ is small enough.

Define $v_{s}=e^{s A} h_{s}$ and by definition, $v_{0}=u$. Then

$$
\partial_{s} v_{s}=(-1)^{K-1} \frac{s^{K-1}}{(K-1) !} e^{s A} A^{K} u+e^{s A} A(\theta-1) \xi_{s}+e^{s A}(\theta-1) \partial_{s} \xi_{s} .
$$

Since the Ornstein-Uhlenbeck is a contraction in $L^{1}(\mathrm{~d} \gamma)$, together with (2.18), we have

$$
\int\left|v_{t}-u\right| \mathrm{d} \gamma \leq C_{K} \int_{0}^{t} \int\left[t^{K-1}\left|A^{K} u\right|+\left|A(\theta-1) \xi_{s}\right|+\left|(\theta-1) \partial_{s} \xi_{s}\right|\right] \mathrm{d} \gamma \mathrm{d} s \leq C_{K} t^{K}
$$

for sufficiently small $t$.

Notice that $h_{t}$ may not be normalized as a probability density w.r.t. $\gamma$ but it is easy to check that there is a constant $c_{t}=1+O\left(t^{M}\right)$, for any $M>0$ positive, such that $c_{t} h_{t}$ is a probability density. Clearly,

$$
\alpha_{t}:=\int x c_{t} h_{t} \mathrm{~d} \gamma=O\left(t^{M}\right), \quad \sigma_{t}^{2}:=\int\left(x-\alpha_{t}\right)^{2} c_{t} h_{t} \mathrm{~d} \gamma=1+O\left(t^{M}\right),
$$

and the same formulas hold if $h_{t}$ is replaced by $v_{t}$ since the OU flow preserves expectation and variance. Let $g_{t}$ be defined by

$$
g_{t}(x) e^{-x^{2} / 2}=c_{t} \sigma_{t}^{-1} h_{t}\left(\left(x+\alpha_{t}\right) \sigma_{t}^{-1}\right) e^{-\left(x+\alpha_{t}\right)^{2} / 2 \sigma_{t}^{2}} .
$$


Then $g_{t}$ is a probability density w.r.t. $\gamma$ with zero mean and variance 1 . It is easy to check that the total variation norm of $h_{t}-g_{t}$ is smaller than any power of $t$. Using again the contraction property of $e^{t A}$ and (4.4), we get

$$
\int\left|e^{t A} g_{t}-u\right| \mathrm{d} \gamma \leq \int\left|e^{t A} g_{t}-e^{t A} h_{t}\right| \mathrm{d} \gamma+\int\left|v_{t}-u\right| \mathrm{d} \gamma \leq C t^{K}
$$

for sufficiently small $t$.

Now we check the LSI constant for $g_{t}$. Recall that $g_{t}$ was obtained from $h_{t}$ by translation and dilation. By definition of the LSI constant, the translation does not change it. The dilation changes the constant, but since our dilation constant is nearly one, the change of LSI constant is also nearly one. So we only have to compare the LSI constants between $\mathrm{d} \nu=u \mathrm{~d} \gamma$ and $c_{t} h_{t} \mathrm{~d} \gamma$. From (4.3) and that $c_{t}$ is nearly one, the LSI constant changes by a factor less than 2 . This proves the claim on the LSI constant.

Finally, the (4.2) directly follows from

$$
\int\left|e^{t \mathcal{A}} G_{t}-F\right| \mathrm{d} \gamma^{\otimes n} \leq N^{2} \int\left|e^{t A} g_{t}-u\right| \mathrm{d} \gamma
$$

and this completes the proof of Proposition 4.1.

We now apply Theorem 2.1 to the initial distribution given by the eigenvalues of the symmetric Wigner ensemble with distribution $g_{\tau} \gamma$ where $\tau=N^{-\zeta}$. By Proposition 4.1, the LSI constant of $g_{\tau} \gamma$ is bounded by the initial LSI constant of $\nu$ by a factor of at most two. Thus we can apply Lemma 2.2 to verify Assumptions 1 and 2 of Theorem 2.1. Assumption 3 follows from the local semicircle law, Theorem 5.1. Thus the correlation functions of the eigenvalues of the ensemble with distribution $\left(e^{\tau A} g_{\tau}\right) \gamma$ are the same as those of GOE in the sense of (2.13). Finally, using (4.2), we can approximate the $k$-point correlation function w.r.t. $\left(e^{\tau A} g_{\tau}\right) \gamma$ by the one w.r.t. $\nu$ after choosing $K$ sufficiently large so that $N^{2} \tau^{K}=N^{2-K \zeta}=o\left(N^{-k}\right)$. The additional smallness factor $N^{-k}$ for the estimate on the total variation in (4.2) is necessary to conclude the convergence of the $k$-point correlation function, since it is rescaled by a factor $N$ in each variable. We also used the trivial fact that the total variation distance of two eigenvalue distributions is bounded by the total variation distance of the distributions of the full matrix ensembles. Finally we remark that the $b \rightarrow 0$ limit in (2.19) is needed to replace $\varrho_{s c}(E)$ in $(2.13)$ with $\varrho_{s c}\left(E^{\prime}\right)$ in $(2.19)$ using the continuity of $O$. This concludes the proof of Theorem 2.3.

Proof of Theorem 2.1. Step 1. The first step is to show that the right hand side of (3.11) vanishes in the large $N$ limit for $\eta=N^{-\varepsilon_{3}}$ with $\varepsilon_{3}$ small enough provided that the estimates (2.10), (2.11) hold. By (2.11), $x_{j} \in I_{j}$ (recall the definition of $I_{j}$ from (3.1)) with a very high probability. In this paper we will say that an event holds with a very high probability if the complement event has a probability that is subexponentially small in $N$, i.e., it is bounded by $C \exp \left(-N^{\varepsilon}\right)$ with some fixed $\varepsilon>0$. From the definition of $b_{j}(3.6)$, we have

$$
b_{j}=\frac{\beta}{N} \sum_{k:|k-j|>N \eta}\left[\frac{\operatorname{sgn}\left(x_{j}-x_{k}\right)}{\left|x_{j}-x_{k}\right|+\eta}-\frac{\operatorname{sgn}\left(x_{j}-\gamma_{k}\right)}{\left|x_{j}-\gamma_{k}\right|+\eta}\right] .
$$

Notice that function $g(x):=\frac{\operatorname{sgn}(\mathrm{x})}{|x|+\eta}$ satisfies

$$
|g(x)-g(y)| \leq \eta^{-2}|x-y|
$$

as long as $x$ and $y$ have the same sign. In our case, $x_{j}-x_{k}$ and $x_{j}-\gamma_{k}$ have the same sign as long as

$$
\left|x_{k}-\gamma_{k}\right|<\left|x_{k}-x_{j}\right|, \quad \text { for all } k \text { satisfying }|k-j|>N \eta \text {. }
$$


The last inequality holds with a very high probability due to $(2.11)$ provided $\varepsilon_{3}$ is smaller than $\mathfrak{b}$. We remark that this is the only place where we used Assumption 2. Thus,, with a very high probability, we have

$$
\left|b_{j}\right| \leq \eta^{-2} \frac{\beta}{N} \sum_{k:|k-j|>N \eta}\left|x_{k}-\gamma_{k}\right|
$$

The contribution to $\Lambda$ of the exceptional event is negligible, since its probability is subexponentially small in $N$ and $\left|b_{j}\right| \leq C \eta \leq C N^{\varepsilon_{3}}$. Thus, recalling the definition of $Q$ from (2.9) and the definition of $\Lambda$ from (3.9), we can bound the error term on the right hand side of (3.11) by

$$
N^{\delta / 2} \Lambda^{1 / 2} \leq C N^{\delta / 2} Q^{1 / 2} \eta^{-2} \leq C N^{-\mathfrak{a}+2 \varepsilon_{3}+\delta / 2} \leq N^{-\mathfrak{a} / 2} \rightarrow 0,
$$

provided that (2.10) holds and $\varepsilon_{3}$ and $\delta$ are small enough, depending on $\mathfrak{a}$.

Step 2. From (3.11) to correlation functions. The equation (3.11) shows that for a special class of observables, depending only on rescaled differences of the points $x_{j}$, the expectations w.r.t. $f_{t} \mu$ and w.r.t the equilibrium measure $\mu$ are identical in the large $N$ limit. But the class of observables in (2.13) of Theorem 2.1 is somewhat bigger and we need to extend our result to them. Without the $\mathrm{d} E^{\prime}$ integration in (2.13), the observable would strongly depend on a fixed energy $E^{\prime}$ and could not be approximated by observables depending only on differences of $x_{j}$. Taking a small averaging in $E^{\prime}$ remedies this problem.

We will consider $E, b$ and $n$ fixed, i.e., the constants in this proof may depend on these three parameters. We start with the identity

$$
\begin{gathered}
\int_{E-b}^{E+b} \frac{\mathrm{d} E^{\prime}}{2 b} \int_{\mathbb{R}^{n}} \mathrm{~d} \alpha_{1} \ldots \mathrm{d} \alpha_{n} O\left(\alpha_{1}, \ldots, \alpha_{n}\right) p_{\tau, N}^{(n)}\left(E^{\prime}+\frac{\alpha_{1}}{N \varrho(E)}, \ldots, E^{\prime}+\frac{\alpha_{n}}{N \varrho(E)}\right) \\
=\int_{E-b}^{E+b} \frac{\mathrm{d} E^{\prime}}{2 b} \int_{i_{1} \neq i_{2} \neq \ldots \neq i_{n}} \widetilde{O}\left(N\left(x_{i_{1}}-E^{\prime}\right), N\left(x_{i_{1}}-x_{i_{2}}\right), \ldots N\left(x_{i_{n-1}}-x_{i_{n}}\right)\right) f_{\tau} \mathrm{d} \mu,
\end{gathered}
$$

where $\widetilde{O}\left(u_{1}, u_{2}, \ldots u_{n}\right):=O\left(\varrho(E) u_{1}, \varrho(E)\left(u_{1}-u_{2}\right), \ldots\right)$. By permutational symmetry of $p_{\tau, N}^{(n)}$ we can assume that $O$ is symmetric and we can restrict the last summation to $i_{1}<i_{2}<\ldots<i_{n}$ upon an overall factor $n$ !. Let $S_{n}$ denote the set of increasing positive integers, $\mathbf{m}=\left(m_{2}, m_{3}, \ldots, m_{n}\right) \in \mathbb{N}_{+}^{n-1}, m_{2}<m_{3}<\ldots<m_{n}$. For a given $\mathbf{m} \in S_{n}$, we change the indices to $i_{1}=i, i_{2}=i+m_{2}, i_{3}=i+m_{3}, \ldots$, and rewrite the sum on the r.h.s. of (4.11) as

$$
\sum_{\mathbf{m} \in S_{n}} \sum_{i=1}^{N} \widetilde{O}\left(N\left(x_{i}-E^{\prime}\right), N\left(x_{i}-x_{i+m_{2}}\right), N\left(x_{i+m_{2}}-x_{i+m_{3}}\right), \ldots\right)=\sum_{\mathbf{m} \in S_{n}} \sum_{i=1}^{N} Y_{i, \mathbf{m}}\left(E^{\prime}, \mathbf{x}\right),
$$

where we introduced

$$
Y_{i, \mathbf{m}}\left(E^{\prime}, \mathbf{x}\right):=\widetilde{O}\left(N\left(x_{i}-E^{\prime}\right), N\left(x_{i}-x_{i+m_{2}}\right), \ldots, N\left(x_{i}-x_{i+m_{n}}\right)\right) .
$$

We will set $Y_{i, \mathbf{m}}=0$ if $i+m_{n}>N$. We have to show that

$$
\lim _{N \rightarrow \infty}\left|\int_{E-b}^{E+b} \frac{\mathrm{d} E^{\prime}}{2 b} \int \sum_{\mathbf{m} \in S_{n}} \sum_{i=1}^{N} Y_{i, \mathbf{m}}\left(E^{\prime}, \mathbf{x}\right) f_{\tau} \mathrm{d} \mu-\int_{E-b}^{E+b} \frac{\mathrm{d} E^{\prime}}{2 b} \int \sum_{\mathbf{m} \in S_{n}} \sum_{i=1}^{N} Y_{i, \mathbf{m}}\left(E^{\prime}, \mathbf{x}\right) \mathrm{d} \mu\right|=0 .
$$


Let $M$ be an $N$-dependent parameter chosen at the end of the proof. Let

$$
S_{n}(M):=\left\{\mathbf{m} \in S_{n}, m_{n} \leq M\right\}, \quad S_{n}^{c}(M):=S_{n} \backslash S_{n}(M),
$$

and note that $\left|S_{n}(M)\right| \leq M^{n-1}$. To prove (4.12), it is sufficient to show that

$$
\lim _{N \rightarrow \infty}\left|\int_{E-b}^{E+b} \frac{\mathrm{d} E^{\prime}}{2 b} \int \sum_{\mathbf{m} \in S_{n}(M)} \sum_{i=1}^{N} Y_{i, \mathbf{m}}\left(E^{\prime}, \mathbf{x}\right) f_{\tau} \mathrm{d} \mu-\int_{E-b}^{E+b} \frac{\mathrm{d} E^{\prime}}{2 b} \int \sum_{\mathbf{m} \in S_{n}(M)} \sum_{i=1}^{N} Y_{i, \mathbf{m}}\left(E^{\prime}, \mathbf{x}\right) \mathrm{d} \mu\right|=0
$$

and that

$$
\lim _{N \rightarrow \infty} \sum_{\mathbf{m} \in S_{n}^{c}(M)}\left|\int_{E-b}^{E+b} \frac{\mathrm{d} E^{\prime}}{2 b} \int \sum_{i=1}^{N} Y_{i, \mathbf{m}}\left(E^{\prime}, \mathbf{x}\right) f_{\tau} \mathrm{d} \mu\right|=0
$$

hold for any $\tau \geq \eta^{1 / 3} N^{\delta}$ (note that $\tau=\infty$ corresponds to the equilibrium, $f_{\infty}=1$ ), where $\eta^{1 / 3} N^{\delta}$ is chosen in Theorem 2.1 and $\eta$ is chosen in the Step 1.

Case 1: Small $\mathbf{m}$ case; proof of (4.13).

After performing the $\mathrm{d} E^{\prime}$ integration, we will eventually apply Theorem 3.1 to the function

$$
G\left(u_{1}, u_{2}, \ldots\right):=\frac{1}{2 b} \int_{\mathbb{R}} \widetilde{O}\left(y, u_{1}, u_{2}, \ldots,\right) \mathrm{d} y,
$$

i.e., to the quantity

$$
\int_{\mathbb{R}} \frac{\mathrm{d} E^{\prime}}{2 b} Y_{i, \mathbf{m}}\left(E^{\prime}, \mathbf{x}\right)=\frac{1}{N} G\left(N\left(x_{i}-x_{i+m_{2}}\right), \ldots\right)
$$

for each fixed $i$ and $\mathbf{m}$.

For any $E$ and $0<\xi<b$ define sets of integers $J=J_{E, b, \xi}$ and $J^{ \pm}=J_{E, b, \xi}^{ \pm}$by

$$
J:=\left\{i: \gamma_{i} \in[E-b, E+b]\right\}, \quad J^{ \pm}:=\left\{i: \gamma_{i} \in[E-(b \pm \xi), E+b \pm \xi]\right\},
$$

where $\gamma_{i}$ was defined in (2.8). Clearly $J^{-} \subset J \subset J^{+}$. With these notations, we have

$$
\int_{E-b}^{E+b} \frac{\mathrm{d} E^{\prime}}{2 b} \sum_{i=1}^{N} Y_{i, \mathbf{m}}\left(E^{\prime}, \mathbf{x}\right)=\int_{E-b}^{E+b} \frac{\mathrm{d} E^{\prime}}{2 b} \sum_{i \in J^{+}} Y_{i, \mathbf{m}}\left(E^{\prime}, \mathbf{x}\right)+\Omega_{J, \mathbf{m}}^{+}(\mathbf{x}) .
$$

The error term $\Omega_{J, \mathbf{m}}^{+}$, defined by (4.16) indirectly, comes from those $i \notin J^{+}$indices, for which $x_{i} \in[E-$ $b, E+b]+O\left(N^{-1}\right)$ since $Y_{i, \mathbf{m}}\left(E^{\prime}, \mathbf{x}\right)=0$ unless $\left|x_{i}-E^{\prime}\right| \leq C / N$, the constant depending on the support of $O$. Thus

$$
\left|\Omega_{J, \mathbf{m}}^{+}(\mathbf{x})\right| \leq C N^{-1} \#\left\{i:\left|x_{i}-\gamma_{i}\right| \geq \xi / 2\right\}
$$

for any sufficiently large $N$ assuming $\xi \gg 1 / N$ and using that $O$ is a bounded function. The additional $N^{-1}$ factor comes from the $\mathrm{d} E^{\prime}$ integration. Taking the expectation with respect to the measure $f_{\tau} \mathrm{d} \mu$, and by a Schwarz inequality, we get

$$
\int\left|\Omega_{J, \mathbf{m}}^{+}(\mathbf{x})\right| f_{\tau} \mathrm{d} \mu \leq C \xi^{-1} N^{-1}\left\{\int\left[\sum_{i=1}^{N}\left|x_{i}-\gamma_{i}\right|\right]^{2} f_{\tau} \mathrm{d} \mu\right\}^{1 / 2}=C \xi^{-1} N^{-1 / 2-\mathfrak{a}}
$$


using Assumption 1 (2.10). We can also estimate

$$
\begin{aligned}
\int_{E-b}^{E+b} \frac{\mathrm{d} E^{\prime}}{2 b} \sum_{i \in J^{+}} Y_{i, \mathbf{m}}\left(E^{\prime}, \mathbf{x}\right) & \leq \int_{E-b}^{E+b} \frac{\mathrm{d} E^{\prime}}{2 b} \sum_{i \in J^{-}} Y_{i, \mathbf{m}}\left(E^{\prime}, \mathbf{x}\right)+C N^{-1}\left|J^{+} \backslash J^{-}\right| \\
& =\int_{\mathbb{R}} \frac{\mathrm{d} E^{\prime}}{2 b} \sum_{i \in J^{-}} Y_{i, \mathbf{m}}\left(E^{\prime}, \mathbf{x}\right)+C N^{-1}\left|J^{+} \backslash J^{-}\right|+\Xi_{J, \mathbf{m}}^{+}(\mathbf{x}) \\
& \leq \int_{\mathbb{R}} \frac{\mathrm{d} E^{\prime}}{2 b} \sum_{i \in J} Y_{i, \mathbf{m}}\left(E^{\prime}, \mathbf{x}\right)+C N^{-1}\left|J^{+} \backslash J^{-}\right|+C N^{-1}\left|J \backslash J^{-}\right|+\Xi_{J, \mathbf{m}}^{+}(\mathbf{x}),
\end{aligned}
$$

where the error term $\Xi_{J, \mathbf{m}}^{+}$, defined by (4.18), comes from indices $i \in J^{-}$such that $x_{i} \notin[E-b, E+b]+O(1 / N)$. It satisfies the same bound (4.17) as $\Omega_{J, \mathbf{m}}^{+}$. By the continuity of $\varrho$, the density of $\gamma_{i}$ 's is bounded by $C N$, thus $\left|J^{+} \backslash J^{-}\right| \leq C N \xi$ and $\left|J \backslash J^{-}\right| \leq C N \xi$. Therefore, summing up the formula (4.15) for $i \in J$, we obtain from (4.16) and (4.18)

$$
\int_{E-b}^{E+b} \frac{\mathrm{d} E^{\prime}}{2 b} \int \sum_{i=1}^{N} Y_{i, \mathbf{m}}\left(E^{\prime}, \mathbf{x}\right) f_{\tau} \mathrm{d} \mu \leq \int \frac{1}{N} \sum_{i \in J} G\left(N\left(x_{i}-x_{i+m_{2}}\right), \ldots\right) f_{\tau} \mathrm{d} \mu+C \xi+C \xi^{-1} N^{-1 / 2-\mathfrak{a}}
$$

for each $\mathbf{m} \in S_{n}$. A similar lower bound can be obtained analogously, and after choosing $\xi=N^{-1 / 4}$, we obtain

$$
\left|\int_{E-b}^{E+b} \frac{\mathrm{d} E^{\prime}}{2 b} \int \sum_{i=1}^{N} Y_{i, \mathbf{m}}\left(E^{\prime}, \mathbf{x}\right) f_{\tau} \mathrm{d} \mu-\int \frac{1}{N} \sum_{i \in J} G\left(N\left(x_{i}-x_{i+m_{2}}\right), \ldots\right) f_{\tau} \mathrm{d} \mu\right| \leq C N^{-1 / 4}
$$

for each $\mathbf{m} \in S_{n}$.

Adding up (4.19) for all $\mathbf{m} \in S_{n}(M)$, we get

$$
\begin{aligned}
\mid \int_{E-b}^{E+b} \frac{\mathrm{d} E^{\prime}}{2 b} & \int \sum_{\mathbf{m} \in S_{n}(M)} \sum_{i=1}^{N} Y_{i, \mathbf{m}}\left(E^{\prime}, \mathbf{x}\right) f_{\tau} \mathrm{d} \mu \\
& -\int \sum_{\mathbf{m} \in S_{n}(M)} \frac{1}{N} \sum_{i \in J} G\left(N\left(x_{i}-x_{i+m_{2}}\right), \ldots\right) f_{\tau} \mathrm{d} \mu \mid \leq C M^{n-1} N^{-1 / 4},
\end{aligned}
$$

and the same estimate holds for the equilibrium, i.e., if we set $\tau=\infty$ in (4.20). Subtracting these two formulas and applying (3.11) from Theorem 3.1 to each summand on the second term in (4.19) and using (4.10), we conclude that

$$
\left|\int_{E-b}^{E+b} \mathrm{~d} E^{\prime} \int \sum_{\mathbf{m} \in S_{n}(M)} \sum_{i=1}^{N} Y_{i, \mathbf{m}}\left(E^{\prime}, \mathbf{x}\right)\left(f_{\tau} \mathrm{d} \mu-\mathrm{d} \mu\right)\right| \leq C M^{n-1}\left(N^{-1 / 4}+N^{-\mathfrak{a} / 2}\right) .
$$

Choosing

$$
M:=N^{\min \{1 / 4, \mathfrak{a} / 2\} / n},
$$

we obtain that (4.21) vanishes as $N \rightarrow \infty$, and this proves (4.13). 
Step 2. Large $\mathbf{m}$ case; proof of (4.14).

For a fixed $y \in \mathbb{R}, \ell>0$, let

$$
\chi(y, \ell):=\sum_{i=1}^{N} \mathbf{1}\left\{x_{i} \in\left[y-\frac{\ell}{N}, y+\frac{\ell}{N}\right]\right\}
$$

denote the number of points in the interval $[y-\ell / N, y+\ell / N]$. Note that for a fixed $\mathbf{m}=\left(m_{2}, \ldots, m_{n}\right)$, we have

$$
\sum_{i=1}^{N}\left|Y_{i, \mathbf{m}}\left(E^{\prime}, \mathbf{x}\right)\right| \leq C \cdot \chi\left(E^{\prime}, \ell\right) \cdot \mathbf{1}\left(\chi\left(E^{\prime}, \ell\right) \geq m_{n}\right) \leq C \sum_{m=m_{n}}^{\infty} m \cdot \mathbf{1}\left(\chi\left(E^{\prime}, \ell\right) \geq m\right),
$$

where $\ell$ denotes the maximum of $\left|u_{1}\right|+\ldots+\left|u_{n}\right|$ in the support of $\widetilde{O}\left(u_{1}, \ldots, u_{n}\right)$.

Since the summation over all increasing sequences $\mathbf{m}=\left(m_{2}, \ldots, m_{n}\right) \in \mathbb{N}_{+}^{n-1}$ with a fixed $m_{n}$ contains at most $m_{n}^{n-2}$ terms, we have

$$
\sum_{\mathbf{m} \in S_{n}^{c}(M)}\left|\int_{E-b}^{E+b} \mathrm{~d} E^{\prime} \int \sum_{i=1}^{N}\right| Y_{i, \mathbf{m}}\left(E^{\prime}, \mathbf{x}\right)\left|f_{\tau} \mathrm{d} \mu\right| \leq C \int_{E-b}^{E+b} \mathrm{~d} E^{\prime} \sum_{m=M}^{\infty} m^{n-1} \int \mathbf{1}\left(\chi\left(E^{\prime}, \ell\right) \geq m\right) f_{\tau} \mathrm{d} \mu .
$$

Now we use Assumption 3 for the interval $I=\left[E^{\prime}-N^{-1+\sigma}, E^{\prime}+N^{-1+\sigma}\right]$ with $\sigma:=\frac{1}{2 n} \min \{1 / 4, \mathfrak{a} / 2\}$. Clearly $\mathcal{N}_{I} \geq \chi\left(E^{\prime}, \ell\right)$ for sufficiently large $N$, thus we get from (2.12) that

$$
\sum_{m=M}^{\infty} m^{n-1} \int \mathbf{1}\left(\chi\left(E^{\prime}, \ell\right) \geq m\right) f_{\tau} \mathrm{d} \mu \leq C_{a} \sum_{m=M}^{\infty} m^{n-1}\left(\frac{m}{N^{\sigma}}\right)^{-a}
$$

holds for any $a \in \mathbb{N}$. By the choice of $\sigma$, we get that $\sqrt{m} \geq N^{\sigma}$ for any $m \geq M$ (see (4.22)), and thus choosing $a=2 n+2$, we get

$$
\sum_{m=M}^{\infty} m^{n-1} \int \mathbf{1}\left(\chi\left(E^{\prime}, \ell\right) \geq m\right) f_{\tau} \mathrm{d} \mu \leq \frac{C_{a}}{M} \rightarrow 0
$$

as $N \rightarrow \infty$. Inserting this into (4.24), this completes the proof of (4.14) and the proof of Theorem 2.1.

\section{$5 \quad$ Local semicircle law and proof of Lemma 2.2}

We first recall the local semicircle law concerning the eigenvalues $x_{1}<x_{2}<\ldots<x_{N}$ of $H$. Let

$$
m(z):=\frac{1}{N} \operatorname{Tr} \frac{1}{H-z}=\frac{1}{N} \sum_{j=1}^{N} \frac{1}{x_{j}-z}
$$

be the Stieltjes transform of the empirical eigenvalue distribution at spectral parameter $z=E+i \eta, \eta>0$, and let

$$
m_{s c}(z):=\int \frac{\varrho_{s c}(x)}{x-z} \mathrm{~d} x
$$

be the Stieltjes transform of the semicirle distribution. In Theorem 4.1 of [11] we proved the following version of the local semicircle law (we remark that, contrary to what is stated in Theorem 4.1 of [11], condition (2.5) of [11] is not needed and has not been used in the proof): 
Theorem 5.1 (Local semicircle law) Assume that the distribution $\nu$ of the matrix elements of the symmetric Wigner matrix ensemble satisfies (2.15) with some constant $\theta$ and assume that $y$ is such that $(\log N)^{4} / N \leq|y| \leq 1$. Then for any $K>0$ there exist positive constants $\delta_{0}, C$ and $c>0$, depending only on $K$ and $\theta$, such that for any $|x| \leq K$ we have

$$
\mathbb{P}\left(\left|m(x+i y)-m_{s c}(x+i y)\right| \geq \delta\right) \leq C e^{-c \delta \sqrt{N|y||2-| x||}},
$$

for all $0 \leq \delta \leq \delta_{0}$ and for all $N$ large enough.

As a corollary of the local semicircle law, the number of eigenvalues up to a fixed energy $E$ can be estimated. The precise statement is the following proposition and it was proven in Proposition 4.2, equation (4.14) of [11].

Proposition 5.2 Assume that the distribution $\nu$ of the matrix elements of the symmetric Wigner matrix ensemble satisfies (2.15) with some finite constant $\theta$. Let

$$
n(E):=\frac{1}{N} \mathbb{E} \#\left\{x_{j} \leq E\right\}
$$

be the expectation of the empirical distribution function of the eigenvalues and recall the definition of $n_{s c}(E)$ from (2.7). Then there exists a constant $C>0$, depending only on the constant $\theta$ in (2.15), such that

$$
\int_{-\infty}^{\infty}\left|n(E)-n_{s c}(E)\right| \mathrm{d} E \leq \frac{C}{N^{6 / 7}} .
$$

The local semicircle law implies that the local density of eigenvalues is bounded, but the estimate in Theorem 5.1 deteriorates near the spectral edges. The following upper bound on the number of eigenvalues in an interval provides a uniform control near the edges. This lemma was essentially proved in Theorem 4.6 of [10] using ideas from an earlier version, Theorem 5.1 of [9]. For the convenience of the reader, a detailed proof is given in the Appendix C.

Lemma 5.3 [Upper bound on the number of eigenvalues] Consider a Wigner matrix with single entry distribution $\nu$ that satisfies the logarithmic Sobolev inequality (2.15) with some constant $\theta$. Let $\mathcal{N}_{I}$ denote the number of eigenvalues in an interval $I \subset \mathbb{R}$. Suppose that $|I| \geq(\log N)^{2} / N$, then there exist positive constants $C, c$ and $K_{0}$, depending only on $\theta$, such that

$$
\mathbb{P}\left(\mathcal{N}_{I} \geq K N|I|\right) \leq C e^{-c \sqrt{K N|I|}}
$$

for any $K \geq K_{0}$.

We remark that for Theorem 5.1 and Lemma 5.3 it is sufficient to assume only the Gaussian decay condition (2.16) instead of the logarithmic Sobolev inequality (2.15).

We can now start to prove Lemma 2.2. Since the logarithmic Sobolev inequality holds for $\nu$ (2.15), it also holds for $\nu_{t}$ as well; for a proof see Lemma B.1 in Appendix B and recall that $\nu_{t}$ is the convolution of $\nu$ with the Ornstein-Uhlenbeck kernel which itself satisfies the logarithmic Sobolev inequality. Moreover, the LSI constant of $\nu_{t}$ is bounded uniformly for all $t>0$, since it is the maximum of the LSI constant $\theta$ of $\nu$ and the LSI constant of the Ornstein-Uhlenbeck kernel, which is bounded uniformly in time. Therefore Lemma 2.2 follows immediately from its time independent version: 
Lemma 5.4 Suppose that the distribution $\nu$ of the matrix elements of the symmetric Wigner ensemble satisfies (2.15) with some finite constant $\theta$. Then there exist positive constants $\mathfrak{a}, \mathfrak{b}$ and $\mathfrak{c}$ such that

$$
\mathbb{E}\left[\frac{1}{N} \sum_{j=1}^{N}\left|x_{j}-\gamma_{j}\right|\right]^{2} \leq N^{-1-2 \mathfrak{a}}
$$

and

$$
\mathbb{P}\left\{\max _{j=1, \ldots, N}\left|x_{j}-\gamma_{j}\right| \geq N^{-\mathfrak{b}}\right\} \leq \exp \left[-N^{\mathfrak{c}}\right]
$$

hold for the eigenvalues $x_{j}$ of $H$ and for any $N \geq N_{0}=N_{0}(\theta, \mathfrak{a}, \mathfrak{b}, \mathfrak{c})$.

The proof of Lemma 5.4 is divided into two steps. In the first step, Section 5.1, we estimate the fluctuation of the eigenvalues $x_{j}$ around their mean values using the logarithmic Sobolev inequality. In the second step, Section 5.2, we estimate the deviation of the mean location of $x_{j}$ from the classical location $\gamma_{j}$ using (5.3).

\subsection{Fluctuation of the eigenvalues around their mean}

Denote by

$$
\alpha_{j}=\mathbb{E} x_{j}
$$

the expected location of $x_{j}$. We start with an estimate on the expected location of the extreme eigenvalues:

Lemma 5.5 Suppose that the probability measure $\nu$ of the matrix entries satisfies

$$
\int_{\mathbb{R}} e^{c_{0}|x|} \mathrm{d} \nu(x)<\infty
$$

for some $c_{0}>0$ (this condition is satisfied, in particular, under (2.15), see (2.16)). Then for any $\delta>0$ we have

$$
-2-C_{0} N^{-1 / 4+\delta} \leq \alpha_{1}<\alpha_{N} \leq 2+C_{0} N^{-1 / 4+\delta}
$$

with some constant $C_{0}$ depending on $\delta$ and $c_{0}$.

Proof. For any $M$, define the probability measure

$$
\zeta_{M}(\mathrm{~d} x):=Z_{M}^{-1} 1(|x| \leq M) \nu(\mathrm{d} x)
$$

on $\mathbb{R}$, where $Z_{M}$ is the normalization factor. Setting $M=N^{\delta}$ and using (5.8), the total variational norm between $\nu$ and $\zeta_{M}$ is bounded

$$
\left\|\zeta_{M}-\nu\right\|_{\infty} \leq C e^{-c N^{\delta}} .
$$

Denote by $\zeta_{M}^{N}=\bigotimes_{i<j} \zeta_{M}\left(\nu^{N}\right.$ resp.) the probability law of the random matrices whose matrix elements are distributed according to $\zeta_{M}$ ( $\nu$ resp.). As usual, we neglect the fact that distribution $\nu$ should be replaced with $\widetilde{\nu}$ for the diagonal elements $i=j$. Since the number of index pairs $i \leq j$ is of order $N^{2}$, the total variational norm between $\zeta_{M}^{N}$ and $\nu^{N}$ is bounded by

$$
\left\|\zeta_{M}^{N}-\nu^{N}\right\|_{\infty} \leq C N^{2} e^{-c N^{\delta}}
$$


From Theorem 1.4 of [29] we obtain that for any $\delta>0$

$$
x_{N} \leq 2+C M^{1 / 2} N^{-1 / 4} \log N \leq 2+N^{-1 / 4+\delta}
$$

holds almost surely w.r.t. $\zeta_{M}^{N}$. It follows from (5.11) that $x_{N}$ is bounded w.r.t. the distribution $\nu^{N}$ as well, up to a subexponentially small probability. To estimate the tail of $x_{N}$ w.r.t. $\nu^{N}$, we use that $\max _{j}\left|x_{j}\right|^{2} \leq \operatorname{Tr} H^{2}$ and the trivial large deviation bound based upon (5.8),

$$
\mathbb{P}_{\nu^{N}}\left(\max _{j}\left|x_{j}\right| \geq K N\right) \leq \mathbb{P}_{\nu^{N}}\left(\sum_{i j}\left|h_{i j}\right|^{2} \geq(K N)^{2}\right) \leq N^{2} \int \mathbf{1}\left(|y|^{2} \geq K^{2} N\right) \mathrm{d} \nu(y) \leq C e^{-c K \sqrt{N}},
$$

that holds for any $K>0$ with constants $C, c$ depending on $c_{0}$. We thus obtain that the expectations of $x_{N}$ w.r.t. these two measures satisfy

$$
\left|\mathbb{E}_{\zeta_{M}^{N}} x_{N}-\mathbb{E}_{\nu^{N}} x_{N}\right| \leq C N^{2} e^{-c N^{\delta}}
$$

From (5.12) we also have

$$
\mathbb{E}_{\zeta_{M}^{N}} x_{N} \leq 2+N^{-1 / 4+\delta}
$$

Thus we have proved that, for any $\delta>0$,

$$
\alpha_{N}=\mathbb{E}_{\nu^{N}} x_{N} \leq 2+C N^{-1 / 4+\delta}
$$

with some constant $C$ depending on $c_{0}$ and $\delta$. Similar lower bound holds for $\alpha_{1}$.

Next we estimate the fluctuations of $x_{j}$ :

Proposition 5.6 For any $\varepsilon>0$ we have

$$
\mathbb{P}\left(\max _{j}\left|x_{j}-\alpha_{j}\right| \geq N^{-1 / 2+\varepsilon}\right) \leq C e^{-c N^{\varepsilon}}
$$

with a constant $C$ depending on $\varepsilon$ and $\theta$.

Proof. First order perturbation theory of the eigenvalue $x_{j}$ of $H$ shows that

$$
\left|\nabla x_{j}\right|^{2}:=\sum_{\ell, k}\left|\frac{\partial x_{j}}{\partial x_{\ell k}}\right|^{2}=\frac{1}{N} \sum_{\ell, k}\left|\frac{\partial x_{j}}{\partial h_{\ell k}}\right|^{2} \leq \frac{C}{N} \sum_{\ell, k}\left|\mathbf{u}_{j}(k) \mathbf{u}_{j}(\ell)\right|^{2}=\frac{C}{N},
$$

where $x_{\ell k}=N^{1 / 2} h_{\ell k}$ are the unscaled random variables, see $(2.14)$, and $\mathbf{u}_{j}=\left(\mathbf{u}_{j}(1), \mathbf{u}_{j}(2), \ldots, \mathbf{u}_{j}(N)\right)$ is the normalized eigenvector belonging to $x_{j}$.

Using (2.15) and the Bobkov-Götze concentration inequality (Theorem 2.1 of [4]), we have for any $T>0$

$$
\mathbb{P}\left(\max _{j}\left|x_{j}-\mathbb{E} x_{j}\right| \geq \gamma\right) \leq 2 N \max _{j} \mathbb{P}\left(x_{j}-\mathbb{E} x_{j} \geq \gamma\right) \leq 2 N e^{-\gamma T} \max _{j} \mathbb{E} e^{\theta T^{2}\left|\nabla x_{j}\right|^{2}} \leq C N e^{-c \gamma^{2} N}
$$

after optimizing for $T$ and using that $\left|\nabla x_{j}\right| \leq C N^{-1 / 2}$ from above. This proves (5.14).

The following proposition is a refinement of Proposition 5.6: 
Proposition 5.7 Fix a sufficiently small constant $\delta>0$ and set $\kappa=N^{-1 / 18+\delta}$. Then for any index $i$ with $C N \kappa^{3 / 2} \leq i \leq N\left(1-C \kappa^{3 / 2}\right)$ we have

$$
\mathbb{P}\left(\left|x_{i}-\alpha_{i}\right| \geq N^{-5 / 9+2 \delta}\right) \leq C e^{-c N^{\delta}}
$$

and

$$
\mathbb{P}\left(\frac{1}{N} \sum_{i=1}^{N}\left|x_{i}-\alpha_{i}\right| \geq N^{-5 / 9+2 \delta}\right) \leq C e^{-c N^{\delta}} .
$$

The constants $C$ and $c$ depend on $\delta$ and $\theta$ but are independent of $N$.

As a preparation for the proof of Proposition 5.7, we need the following estimate on the tail of the gap distribution.

Lemma 5.8 Let $|E|<2$. Denote by $x_{\alpha}$ the largest eigenvalue below $E$ and assume that $\alpha \leq N-1$. Then there exist positive constants $C$ and $c$, depending only on the Sobolev constant $\theta$ in (2.15), such that for any $M$ that satisfy $c(\log N)^{4} /(2-|E|) \leq M \leq C N(2-|E|)$, we have

$$
\mathbb{P}\left(x_{\alpha+1}-E \geq \frac{M}{N}, \alpha \leq N-1\right) \leq C e^{-c[2-|E|]^{3 / 2} \sqrt{M}} .
$$

This lemma was proven in Theorem E1 of [11], see also Theorem 3.3 of [10], and the proof will not be repeated here. We only mention the main idea, that the local semicircle law, Theorem 5.1, provides a positive lower bound on the number of eigenvalues in any interval $I$ of size $|I| \geq A(\log N)^{4} /[N|2-| x||]$ around the point $x$ with a sufficiently large constant $A$. In particular, it follows that there is at least one eigenvalue in each such interval $I$ with a very high probability.

Proof of Proposition 5.7. We choose $M, K$ positive numbers, depending on $N$, such that

$$
M \kappa^{6} \geq N^{2 \delta}, \quad K \leq c N \kappa^{3 / 2} \text { and } c(\log N)^{4} / \kappa \leq M \leq C N \kappa
$$

with some sufficiently small $c>0$ and large $C>0$ constants. Let

$$
\Phi:=2 N^{-1 / 2+2 \delta} K^{-1 / 2}+\frac{2 K M}{N} .
$$

Consider an index $i$ with $C N \kappa^{3 / 2} \leq i \leq N\left(1-C \kappa^{3 / 2}\right)$, then $|2-| \gamma_{i}|| \geq C \kappa$. We first show that $|2-| x_{i}|| \geq$ $C \kappa^{2}$ with a very high probability. Suppose, in the contrary, that $x_{i}<-2+C \kappa^{2}$ for some $i \geq C N \kappa^{3 / 2}$ (the case $x_{i} \geq 2-C \kappa^{2}$ is treated analogously). From (5.9) and (5.14) it follows that $x_{1} \geq-2-C_{0} N^{-1 / 4+\delta}$ with a very high probability. But then the interval $\left[-2-C_{0} N^{-1 / 4+\delta},-2+C \kappa^{2}\right]$ of length $C_{0} N^{-1 / 4+\delta}+C \kappa^{2} \ll \kappa^{3 / 2}$ would contain $C N \kappa^{3 / 2}$ eigenvalues, an event with an extremely low probability by (5.4).

Knowing that $|2-| x_{i}|| \geq \kappa^{2}$ with a very high probability, we can use (5.17) to conclude that for any index $i$ with $C N \kappa^{3 / 2} \leq i \leq N\left(1-C \kappa^{3 / 2}\right)$ we have

$$
\mathbb{P}\left(x_{i+1}-x_{i} \geq \frac{M}{N}\right) \leq e^{-c \sqrt{M \kappa^{6}}}+C e^{-c N^{\delta}} \leq C e^{-c N^{\delta}}
$$

by (5.18). Then

$$
\mathbb{P}\left(\left|x_{i}-\frac{1}{2 K+1} \sum_{|j-i| \leq K} x_{j}\right| \geq \frac{K M}{N}\right) \leq C e^{-c N^{\delta}}
$$


Similarly to the calculation in Theorem 3.1 of [9], by using the logarithmic Sobolev inequality (2.15), we have

$$
\mathbb{P}\left(\left|\frac{1}{2 K+1} \sum_{j:|j-i| \leq K} x_{j}-\frac{1}{2 K+1} \sum_{j:|j-i| \leq K} \mathbb{E} x_{j}\right| \geq N^{-1 / 2+2 \delta} K^{-1 / 2}\right) \leq C e^{-c N^{\delta}} .
$$

This bound holds for any index $i$ with the remark that if $i<K$ or $i>N-K$, then the averaging over the indices $j$ is done asymmetrically.

Combining this estimate with (5.21) we have, apart from a set of very small probability, that

$$
\left|x_{i}-\frac{1}{2 K+1} \sum_{j:|j-i| \leq K} \alpha_{j}\right| \leq \frac{\Phi}{2}
$$

for any $C N \kappa^{3 / 2} \leq i \leq N\left(1-C \kappa^{3 / 2}\right)$. Taking expectation, and using the tail estimate (5.13) to control $x_{N}$ on the event of very small probability where (5.23) may not hold, we also obtain for these $i$ indices that

$$
\left|\alpha_{i}-\frac{1}{2 K+1} \sum_{j:|j-i| \leq K} \alpha_{j}\right| \leq \frac{\Phi}{2}
$$

Subtracting the last two inequalities yields

$$
\mathbb{P}\left(\left|x_{i}-\alpha_{i}\right| \geq \Phi\right) \leq C e^{-c N^{\delta}}
$$

and combining this bound with the estimate (5.14) for the extreme indices, we obtain

$$
\mathbb{P}\left(\frac{1}{N} \sum_{i=1}^{N}\left|x_{i}-\alpha_{i}\right| \geq C \kappa^{3 / 2} N^{-1 / 2+\delta}+\Phi\right) \leq C e^{-c N^{\delta}}
$$

The inequalities (5.15) and (5.16) now follow if we choose the parameters as

$$
\kappa=N^{-1 / 18+\delta}, \quad M=N^{1 / 3}, \quad K=N^{1 / 9},
$$

which choice is compatible with the conditions (5.18).

\subsection{Deviation of the eigenvalues from their classical locations}

The next Proposition 5.9 below estimates the distance of the eigenvalues from their location given by the semicircle law. This will justify that the convex extension of the potential $W_{j}$ affects only regimes of very small probability.

Proposition 5.9 For any small $\delta>0$ and for any $j=1,2, \ldots N$ we have

$$
\mathbb{P}\left(\left|x_{j}-\gamma_{j}\right| \geq C N^{-1 / 5+\delta}\right) \leq C e^{-c N^{\delta}}
$$

and we also have

$$
\max _{m}\left|\alpha_{m}-\gamma_{m}\right| \leq C N^{-1 / 5+\delta} .
$$

The constants $C$ and $c$ depend on $\delta$ and $\theta$ but are independent of $N$. 
We remark that in the bulk $\alpha_{m}-\gamma_{m}$ is expected to be bounded by $O\left(N^{-1+\varepsilon}\right)$ (in the hermitian case it was proven in [19], see also [28]); near the edges one expects $\alpha_{m}-\gamma_{m} \sim O\left(N^{-2 / 3}\right)$. Our estimate is not optimal, but it gives a short proof that is sufficient for our purpose. We remark that after submitting this paper, these conjectures were proven in [17].

Proof of Proposition 5.9. We define

$$
\widetilde{n}(E):=\frac{1}{N} \#\left\{\alpha_{j} \leq E\right\}
$$

to be the counting function of the expected locations of the eigenvalues. We compare $\widetilde{n}(E)$ with $n(E)$ defined in (5.2). Using the fluctuation bound (5.14), we have

$$
n\left(E-N^{-1 / 2+\varepsilon}\right)-C e^{-c N^{\varepsilon}} \leq \widetilde{n}(E) \leq n\left(E+N^{-1 / 2+\varepsilon}\right)+C e^{-c N^{\varepsilon}}
$$

for any $E \in \mathbb{R}$. In fact, the upper bound on the density (5.4) guarantees that $\widetilde{n}(E)$ and $n(E)$ are Lipschitz continuous on any scale much bigger than $(\log N)^{2} / N$, i.e.

$$
|\widetilde{n}(E)-n(E)| \leq C N^{-1 / 2+\varepsilon}+C e^{-c N^{\varepsilon}} \leq C N^{-1 / 2+\varepsilon}
$$

for any $E$.

We write

$$
\sum_{j=1}^{N}\left|\alpha_{j}-\gamma_{j}\right|=\sum_{j: \alpha_{j} \geq \gamma_{j}}\left(\alpha_{j}-\gamma_{j}\right)+\sum_{j: \alpha_{j} \leq \gamma_{j}}\left(\gamma_{j}-\alpha_{j}\right) .
$$

For the first term

$$
\begin{aligned}
\sum_{j: \alpha_{j} \geq \gamma_{j}}\left(\alpha_{j}-\gamma_{j}\right) & =\int \mathrm{d} E \sum_{j: \alpha_{j} \geq \gamma_{j}} \mathbf{1}\left(\gamma_{j} \leq E \leq \alpha_{j}\right)=\int \mathrm{d} E \sum_{j: \alpha_{j} \geq \gamma_{j}} \mathbf{1}\left(\widetilde{n}(E) \geq \frac{j}{N}>n_{s c}(E)\right) \\
& =N \int \mathrm{d} E \mathbf{1}\left(n_{s c}(E) \leq \widetilde{n}(E)\right)\left(n_{s c}(E)-\widetilde{n}(E)\right)
\end{aligned}
$$

and the second term is analogous. We thus have

$$
\frac{1}{N} \sum_{j=1}^{N}\left|\alpha_{j}-\gamma_{j}\right|=\int\left|\widetilde{n}(E)-n_{s c}(E)\right| \mathrm{d} E .
$$

By Lemma 5.5

$$
\int_{|E| \geq 3}\left|\widetilde{n}(E)-n_{s c}(E)\right| \mathrm{d} E=0 .
$$

For the energy range $|E| \leq 3$, we use (5.28):

$$
\begin{aligned}
\int_{|E| \leq 3}\left|\widetilde{n}(E)-n_{s c}(E)\right| \mathrm{d} E & \leq C N^{-1 / 2+\varepsilon}+\int_{|E| \leq 3}\left|n(E)-n_{s c}(E)\right| \mathrm{d} E \\
& \leq C N^{-1 / 2+\varepsilon}
\end{aligned}
$$


from (5.3). Thus we obtain from (5.30)

$$
\frac{1}{N} \sum_{j=1}^{N}\left|\alpha_{j}-\gamma_{j}\right| \leq C N^{-1 / 2+\varepsilon} .
$$

with an $\varepsilon$ dependent constant.

To estimate $\left|\alpha_{m}-\gamma_{m}\right|$, we can assume, without loss of generality, that $\alpha_{m} \geq \gamma_{m}$, the other case is treated analogously. Let $\lambda>0$ be a parameter that will be optimized later. Set $m_{0}=\left[C N \lambda^{3 / 2}\right]$ with a sufficiently large constant $C$. Since $n_{s c}(-2+\delta) \sim \delta^{3 / 2}$, for any small $\delta>0$, the parameter $\lambda$ is roughly the energy difference from the edge to the $m_{0}$-th eigenvalue.

First we consider an index $m$ such that $m_{0} \leq m \leq N-m_{0}$. For a small positive number $\ell$, define

$$
S:=\left\{j: \gamma_{j} \in\left[\gamma_{m}, \gamma_{m}+\ell\right]\right\} .
$$

From the property $n_{s c}(-2+\delta) \sim \delta^{3 / 2}$ for small $\delta$, we have

$$
|S| \geq c N \ell \lambda^{1 / 2} \text {. }
$$

Now set $\ell=\min \left\{\frac{1}{2}\left|\alpha_{m}-\gamma_{m}\right|, c \lambda\right\}$ with some small positive constant $c$. Since for all $j \in S$

$$
\alpha_{j}-\gamma_{j} \geq \alpha_{m}-\left(\gamma_{m}+\ell\right) \geq \frac{1}{2}\left(\alpha_{m}-\gamma_{m}\right) \geq \ell,
$$

we have

$$
\sum_{j=1}^{N}\left|\alpha_{j}-\gamma_{j}\right| \geq \sum_{j \in S} \ell \geq c N \ell^{2} \lambda^{1 / 2} .
$$

Combining this estimate with (5.32), we have

$$
\ell \leq C \lambda^{-1 / 4} N^{-1 / 4+\varepsilon / 2} .
$$

Assuming that $\lambda \geq C N^{-1 / 5+2 \varepsilon / 5}$, we see that $\ell=\frac{1}{2}\left|\alpha_{m}-\gamma_{m}\right|$ and we obtain

$$
\left|\alpha_{m}-\gamma_{m}\right| \leq C N^{-1 / 5+\varepsilon}
$$

for any $m$ with $m_{0} \leq m \leq N-m_{0}$.

For the extreme indices, we use that if $m \leq m_{0}$, then from Lemma 5.5 and (5.33), we have

$$
-2-C N^{-1 / 4+\delta} \leq \alpha_{1} \leq \alpha_{m} \leq \alpha_{m_{0}} \leq \gamma_{m_{0}}+C N^{-1 / 5+\varepsilon} \leq-2+C \lambda+C N^{-1 / 5+\varepsilon}
$$

and

$$
-2 \leq \gamma_{m} \leq-2+C \lambda
$$

for all $m \leq m_{0}$. Thus

$$
\left|\alpha_{m}-\gamma_{m}\right| \leq C \lambda+C N^{-1 / 5+\varepsilon}
$$

with $C$ depending on $\varepsilon$. Similar estimates hold at the upper edge of the spectrum, i.e. for $m \geq N-m_{0}$. Choosing $\lambda=C N^{-1 / 5+\varepsilon}$, we conclude the proof of (5.27). The proof of (5.26) then follows from (5.14) and this concludes the proof of Proposition 5.9.

The following Proposition is a strengthening of the bound (5.32) used previously. 
Proposition 5.10 With the previous notations, we have

$$
\frac{1}{N} \sum_{i}\left|\alpha_{i}-\gamma_{i}\right| \leq C N^{-5 / 9+2 \delta}
$$

for any $\delta>0$ and with a constant $C$ depending on $\delta$ and $\theta$.

Proof. Recalling the definition of $\Phi$ from (5.19), we will prove that

$$
\frac{1}{N} \sum_{i}\left|\alpha_{i}-\gamma_{i}\right| \leq C \Phi+C \kappa N^{-1 / 2+\delta}
$$

which gives (5.34) with the choice of parameters (5.25). We proceed similarly to the proof of Proposition 5.9 but we notice that in addition to (5.28), a stronger bound on $|n(E)-\widetilde{n}(E)|$ is available for $E \in I:=\left[E_{-}, E_{+}\right]$, where $E_{ \pm}:= \pm\left(2-C_{2} \kappa\right)$, with some large constant $C_{2}$ and setting $\kappa:=N^{-1 / 18+\delta}$ as in Proposition 5.7. To obtain an improved bound, note that for any $E$ in this interval

$$
n(E)=\frac{1}{N} \sum_{j} \mathbb{E} \mathbf{1}\left(x_{j} \leq E\right) \leq \frac{1}{N} \sum_{j} \mathbb{E} \mathbf{1}\left(\alpha_{j} \leq E+\Phi\right)+C e^{-c N^{\delta}}=\widetilde{n}(E+\Phi)+C e^{-c N^{\delta}} .
$$

To see this inequality, define the random index

$$
j_{0}=j_{0}(E):=\max \left\{j: x_{j} \leq E\right\}=\sum_{j} \mathbf{1}\left(x_{j} \leq E\right)
$$

and the deterministic index

$$
j_{1}=j_{1}(E):=\max \left\{j: \alpha_{j} \leq E+\Phi\right\}=\sum_{j} \mathbf{1}\left(\alpha_{j} \leq E+\Phi\right) .
$$

The estimate (5.36) will then follow if we prove that $j_{0} \leq j_{1}$, i.e. $\alpha_{j_{0}} \leq E+\Phi$, with a very high probability. By (5.26) we have, with a very high probability, that

$$
\gamma_{j_{0}}-C N^{-1 / 5+\delta} \leq x_{j_{0}} \leq E \leq x_{j_{0}+1} \leq \gamma_{j_{0}+1}+C N^{-1 / 5+\delta} \leq \gamma_{j_{0}}+C N^{-1 / 5+\delta} .
$$

Therefore, with a very high probability, $\gamma_{j_{0}}$ is in the $C N^{-1 / 5+\delta}$ vicinity of $E \in I$, and thus $C N \kappa^{3 / 2} \leq j_{0} \leq$ $N\left(1-C \kappa^{3 / 2}\right)$ holds for any fixed $C$ if $C_{2}$ in the definition of $E_{ \pm}$is sufficiently large. Thus $\left|x_{j_{0}}-\alpha_{j_{0}}\right| \leq \Phi$ with a very high probability by (5.15), so $x_{j_{0}} \leq E$ implies $\alpha_{j_{0}} \leq E+\Phi$ and this proves (5.36).

The proof of the lower bound

$$
n(E) \geq \widetilde{n}(E-\Phi)-C e^{-c N^{\delta}}
$$

is analogous. Finally, by the Lipschitz continuity of $\widetilde{n}(E)$ on a scale bigger than $(\log N)^{2} / N$, we have

$$
|n(E)-\widetilde{n}(E)| \leq C \Phi, \quad \forall E \in\left[E_{-}, E_{+}\right],
$$

where we also used that $\Phi \geq C \exp \left(-c N^{\delta}\right)$.

Define the interval $J=\left[-2-C_{1} N^{-1 / 4+\delta}, 2+C_{1} N^{1 / 4+\delta}\right]$ with a constant $C_{1}$ larger than the constant $C_{0}$ in (5.9). Using (5.30), we have

$$
\frac{1}{N} \sum_{j=1}^{N}\left|\alpha_{j}-\gamma_{j}\right| \leq(I)+(I I)+(I I I)+\int\left|n(E)-n_{s c}(E)\right| \mathrm{d} E
$$


with

$$
(I):=\int_{I}|\widetilde{n}(E)-n(E)| \mathrm{d} E, \quad(I I):=\int_{J \backslash I}|\widetilde{n}(E)-n(E)| \mathrm{d} E, \quad(I I I):=\int_{J^{c}}|\widetilde{n}(E)-n(E)| \mathrm{d} E .
$$

From (5.37) and (5.28), we have

$$
(I) \leq C \Phi, \quad(I I) \leq C N^{-1 / 2+\delta}|J \backslash I| \leq C \kappa N^{-1 / 2+\delta}
$$

since $|J \backslash I| \leq C \kappa+N^{-1 / 4+\delta} \leq C \kappa$. Finally, $\widetilde{n}(E) \equiv 0$ for $E<-2-C_{0} N^{-1 / 4+\delta}$ and $\widetilde{n}(E) \equiv 1$ for $E>2+C_{0} N^{-1 / 4+\delta}$ by (5.9). Since $C_{1}>C_{0}$, combining these estimates with the fluctuation (5.14) and with the tail estimate (5.13), we obtain that $n(E)(1-n(E)) \leq C \exp \left[-c N^{1 / 4}\right]$ for any $E \in J^{c}$, and it decays exponentially for large $|E|$, therefore

$$
(I I I) \leq C e^{-c N^{1 / 4}}
$$

Collecting all these estimates, inserting them into (5.38) and using (5.3), we obtain (5.35) and conclude the proof of Proposition 5.10.

Finally, we can complete the proof of Lemma 5.4. By (5.16) and (5.34), we have

$$
\frac{1}{N} \sum_{k}\left|x_{k}-\gamma_{k}\right| \leq \frac{1}{N} \sum_{k}\left[\left|\alpha_{k}-\gamma_{k}\right|+\left|x_{k}-\alpha_{k}\right|\right] \leq C N^{-5 / 9+2 \delta}
$$

apart from a set of probability $C \exp \left[-c N^{\delta}\right]$. Combining it with the tail estimate $(5.13)$ on $\max \left|x_{k}\right|$, we obtain (5.5) with any $\mathfrak{a}<1 / 18$. The inequality (5.6) in Lemma 5.4 follows immediately from (5.26) with any $\mathfrak{c}>0$ sufficiently small and with $\mathfrak{b}<1 / 5-\mathfrak{c}$.

\section{A Some Properties of the Eigenvalue Process}

In the main part of the paper we did not specify the function spaces in which the equations (2.4) and (3.12) are solved. In this appendix we summarize some basic properties of these equations. In particular, we justify the integration by parts in (3.17). For simplicity, we consider the most singular $\beta=1$ case only.

The Dyson Brownian motion as a stochastic process was rigorously constructed in Section 4.3.1 of [18]. It was proved that the eigenvalues do not collide with probability one and thus (2.4) holds in a weak sense on the open set $\Sigma_{N}$. The coefficients of $L$ have a $\left(x_{i}-x_{j}\right)^{-1}$ singularity near the coalescence hyperspace $x_{i}=x_{j}$. We focus only on the single collision singularities, i.e. on the case $j=i \pm 1$. By the ordering of the eigenvalues, higher order collision points form a zero measure set on the boundary of $\Sigma_{N}$ and can thus be neglected. In an open neighborhood near the coalescence hyperspace $x_{i}=x_{i+1}$, the generator has the form

$$
L=\frac{1}{2 N}\left(\partial_{i}^{2}+\partial_{i+1}^{2}+\frac{1}{x_{i+1}-x_{i}}\left(\partial_{i+1}-\partial_{i}\right)\right)+L_{r e g}=\frac{1}{4 N}\left(\partial_{v}^{2}+\partial_{u}^{2}+\frac{1}{u} \partial_{u}\right)+L_{r e g}, \quad u>0,
$$

after a change of variables, $v=\frac{1}{2}\left(x_{i}+x_{i+1}\right), u=\frac{1}{2}\left(x_{i+1}-x_{i}\right)$, where $L_{r e g}$ has regular coefficients. The boundary condition at $u=0$ is given by the standard boundary condition of the generator of the Bessel process, $\partial_{u}^{2}+\frac{1}{u} \partial_{u}$, which is $u f^{\prime}(u) \rightarrow 0$ as $u \rightarrow 0+$. Thus $L$ is defined on functions $f \in C^{2}\left(\Sigma_{N}\right)$ with sufficient decay at infinity and with boundary conditions

$$
\lim _{x_{i+1}-x_{i} \rightarrow 0}\left(x_{i+1}-x_{i}\right)\left(\partial_{i+1}-\partial_{i}\right) f \rightarrow 0
$$


for each $i$.

The generator $\widetilde{L}$ of (3.12) differs from $L$ only in drift terms with bounded coefficients, hence the boundary conditions of $\widetilde{L}$ and $L$ coincide. Finally, we need some non-vanishing and regularity property of the solution of $(3.12)$ :

Lemma A.1 Let $\Omega \subset \Sigma^{N}$ be a bounded open set such that

$$
\bar{\Omega} \cap \bigcup_{i<j:(i, j) \neq(1,2)}\left\{\mathbf{x}: x_{i}=x_{j}\right\}=\emptyset
$$

i.e. $\bar{\Omega}$ intersects at most one of the coalescent hyperplanes, namely the $\left\{x_{1}=x_{2}\right\}$. Then any weak solution $q_{t}(\mathbf{x})$ of (3.12) with boundary conditions (A.1) is $C^{2}$ on $(t, \mathbf{x}) \in R_{+} \times \bar{\Omega}$ and for any $t>0$ we have

$$
0<\inf _{\bar{\Omega}} q_{t} \leq \sup _{\bar{\Omega}} q_{t}<\infty
$$

Proof. The statement follows from regularity properties of the Bessel process with generator $\partial_{u}^{2}+\frac{1}{u} \partial_{u}$. In a small neighborhood of the coalescence line $x_{1}=x_{2}$ one can introduce a local coordinate system $(u, \mathbf{y})=$ $\Phi(\mathbf{x})$, where $u=\frac{1}{2}\left(x_{2}-x_{1}\right)>0, \mathbf{y} \in \mathbb{R}^{N-1}$, so that, in the case for GOE,

$$
\widetilde{L}=\frac{1}{4 N}\left[\partial_{u}^{2}+\frac{1}{u} \partial_{u}\right]+L_{r e g},
$$

where $L_{r e g}$ is an elliptic operator with second derivatives in the $\mathbf{y}$ variables and with bounded coefficients on the compact set $\Phi(\bar{\Omega})$. The solution in the new coordinates is $\widetilde{q}_{t}(u, \mathbf{y})=q_{t}\left(\Phi^{-1}(u, \mathbf{y})\right)$. Introducing a function $\widehat{q}_{t}(a, b, \mathbf{y}):=\widetilde{q}_{t}\left(\sqrt{a^{2}+b^{2}}, \mathbf{y}\right)$ defined in $N+1$ variables, we see that $\widehat{q}_{t}$ satisfies $\partial_{t} \widehat{q}_{t}=\widehat{L} \widehat{q}_{t}$, where

$$
\widehat{L}=\frac{1}{N}\left[\partial_{a}^{2}+\partial_{b}^{2}\right]+L_{r e g}
$$

i.e. $\widehat{L}$ is elliptic with bounded coefficients in the new variables. Notice that the boundary condition (A.1) implies that, in the two dimensional plane of $(a, b)$, the support of the test function for the equation $\partial_{t} \widehat{q}_{t}=\widehat{L} \widehat{q}_{t}$ is allowed to include the origin $(0,0)$.

By standard parabolic regularity, we obtain that the solution is $C^{2}$ and is bounded from above and below.

This lemma justifies the integration by parts in (3.17). Since $q \in C^{2}$ and it is separated away from zero, $h=\sqrt{q}$ has no singularity on the coalescence lines. Since the function $\exp (-\widetilde{\mathcal{H}})$ vanishes whenever $x_{i}=x_{j}$ for some $i \neq j$, the boundary terms of the form

$$
\int_{x_{i}=x_{j}} \partial \sqrt{q} \partial^{2} \sqrt{q} e^{-\widetilde{\mathcal{H}}} \mathrm{d} \mathbf{x}
$$

in the integration by parts vanish. 


\section{B Logarithmic Sobolev inequality for convolution measures}

Lemma B.1 Suppose $K$ and $H$ are two probability densities on $\mathbb{R}$ so that the logarithmic Sobolev inequality holds with constants a and $b$, respectively. Then logarithmic Sobolev inequality holds for their convolution $K * H$ as

$$
\int_{\mathbb{R}} f(x) \log f(x) K * H(x) \mathrm{d} x \leq \max (a, b) \int_{\mathbb{R}}(\nabla \sqrt{f(x)})^{2} K * H(x) \mathrm{d} x
$$

for any $f$ with $\int f(x) K * H(x) \mathrm{d} x=1$. Here $\nabla=\mathrm{d} / \mathrm{d} x$.

Proof. The following proof is really a special case of the martingale approach used in [23] to prove LSI. Let

$$
g(y)=\int_{\mathbb{R}} f(x) K(x-y) \mathrm{d} x .
$$

Then the left side of (B.1) is equal to

$$
\iint_{\mathbb{R}}[f(x) \log [f(x) / g(y)]+f(x) \log g(y)] K(x-y) \mathrm{d} x H(y) \mathrm{d} y .
$$

For any fixed $y$, from the LSI w.r.t. the measure $K(x-y) \mathrm{d} x$, the first term on the right hand side is bounded by

$$
a \int_{\mathbb{R}} \int_{\mathbb{R}}(\nabla \sqrt{f(x)})^{2} K(x-y) \mathrm{d} x H(y) \mathrm{d} y .
$$

Since $\int_{\mathbb{R}} g H=1$, the second term in (B.2) is estimated by

$$
\int_{\mathbb{R}} g(y) \log g(y) H(y) \mathrm{d} y \leq b \int_{\mathbb{R}}(\nabla \sqrt{g(y)})^{2} H(y) \mathrm{d} y=\frac{b}{4} \int_{\mathbb{R}} g(y)^{-1}\left(\int_{\mathbb{R}} f(x) \nabla_{y} K(x-y) \mathrm{d} x\right)^{2} H(y) \mathrm{d} y .
$$

Integrating by parts, we can rewrite the last term as

$$
\frac{b}{4} \int_{\mathbb{R}} g(y)^{-1}\left(\int f^{\prime}(x) K(x-y) \mathrm{d} x\right)^{2} H(y) \mathrm{d} y \leq b \int_{\mathbb{R}} \int_{\mathbb{R}}(\nabla \sqrt{f(x)})^{2} K(x-y) \mathrm{d} x H(y) \mathrm{d} y,
$$

where we have used $f^{\prime}(x)=2 \sqrt{f(x)} \nabla \sqrt{f(x)}$ and the Schwarz inequality. Combining these inequalities, we have proved the Lemma.

\section{Proof of Lemma 5.3}

For any $1 \leq k \leq N$, let $H^{(k)}$ denote the $(N-1) \times(N-1)$ minor that is obtained from the Wigner matrix $H$ by removing the $k$-th row and column. Let $\mathbf{a}^{(k)}=\left(h_{k 1}, h_{k 2}, \ldots h_{k, k-1}, h_{k, k+1}, \ldots h_{k N}\right)^{t}$ be the $k$-th column of $H$ without the $h_{k k}$ element. Let $\lambda_{1}^{(k)}<\lambda_{2}^{(k)}<\ldots<\lambda_{N-1}^{(k)}$ be the eigenvalues and $\mathbf{u}_{1}^{(k)}, \mathbf{u}_{2}^{(k)}, \ldots$ the corresponding eigenvectors of $H^{(k)}$ and set

$$
\xi_{\alpha}^{(k)}:=N\left|\mathbf{a}^{(k)} \cdot \mathbf{u}_{\alpha}^{(k)}\right|^{2}, \quad \alpha=1,2, \ldots, N-1 .
$$

It is well known (see, e.g. Lemma 2.5 of [9]), that the eigenvalues of $H^{(k)}$ and $H$ are interlaced for each $k$, i.e.

$$
x_{1}<\lambda_{1}^{(k)}<x_{2}<\lambda_{2}^{(k)}<\ldots<x_{N-1}<\lambda_{N-1}^{(k)}<x_{N} .
$$


Expressing the resolvent $G=(H-z)^{-1}$ of $H$ at a spectral parameter $z=E+i \eta, \eta>0$, in terms of the resolvent of $H^{(k)}$, we obtain

$$
G_{z}(k, k)=\frac{1}{h_{k k}-z-\mathbf{a}^{(k)} \cdot\left(H^{(k)}-z\right)^{-1} \mathbf{a}^{(k)}}=\left[h_{k k}-z-\frac{1}{N} \sum_{\alpha=1}^{N-1} \frac{\xi_{\alpha}^{(k)}}{\lambda_{\alpha}^{(k)}-z}\right]^{-1} .
$$

By considering only the imaginary part, we obtain

$$
\left|G_{z}(k, k)\right| \leq \eta^{-1}\left|1+\frac{1}{N} \sum_{\alpha=1}^{N-1} \frac{\xi_{\alpha}^{(k)}}{\left(\lambda_{\alpha}^{(k)}-E\right)^{2}+\eta^{2}}\right|^{-1} .
$$

For the interval $I \subset \mathbb{R}$ given in Lemma 5.3 , set $E$ to be its midpoint and $\eta:=|I|$, i.e. $I=\left[E-\frac{\eta}{2}, E+\frac{\eta}{2}\right]$. Clearly

$$
\mathcal{N}_{I} \leq C \sum_{j=1}^{N} \frac{\eta^{2}}{\left(x_{j}-E\right)^{2}+\eta^{2}}=\frac{C \eta}{\pi} \operatorname{Im} \operatorname{Tr} G(z)
$$

thus from (C.3) we obtain

$$
\mathcal{N}_{I} \leq C \eta \sum_{k=1}^{N}\left|G_{z}(k, k)\right| \leq C N \eta^{2} \sum_{k=1}^{N}\left|\sum_{\alpha: \lambda_{\alpha}^{(k)} \in I} \xi_{\alpha}^{(k)}\right|^{-1},
$$

where we restricted the $\alpha$ summation in (C.3) only to eigenvalues lying in $I$.

For each $k=1,2, \ldots N$, we define the event

$$
\Omega_{k}:=\left\{\sum_{\alpha: \lambda_{\alpha}^{(k)} \in I} \xi_{\alpha}^{(k)} \leq \delta\left(\mathcal{N}_{I}-1\right)\right\}
$$

for some small $\delta>0$. By the interlacing property of the $\mu_{\alpha}$ and $\lambda_{\alpha}^{(k)}$ eigenvalues, we know that there are at least $\mathcal{N}_{I}-1$ eigenvalues of $H^{(k)}$ in $I$. Since the logarithmic Sobolev inequality (2.15) implies that the tail of the distribution $\nu$ has a Gaussian bound [22], we can apply Lemma C.1 below to conclude that there exists a positive universal constant $c$ such that $\mathbb{P}\left(\Omega_{k}\right) \leq \mathbb{E} \exp \left[-c \sqrt{\mathcal{N}_{I}-1}\right]$. Setting $\widetilde{\Omega}=\bigcup_{k=1}^{N} \Omega_{k}$, we see that

$$
\mathbb{P}\left(\widetilde{\Omega} \text { and } \mathcal{N}_{I} \geq K N|I|\right) \leq N \mathbb{E}\left[e^{-c \sqrt{\mathcal{N}_{I}-1}} \cdot \mathbf{1}\left(\mathcal{N}_{I} \geq K N|I|\right)\right] \leq e^{-c^{\prime} \sqrt{K N|I|}}
$$

if $K$ is sufficiently large, recalling that $\eta=|I| \geq(\log N)^{2} / N$. On the complement event, $\widetilde{\Omega}^{c}$, we have from (C.4) that

$$
\mathcal{N}_{I} \leq \frac{C N^{2} \eta^{2}}{\delta\left(\mathcal{N}_{I}-1\right)}
$$

i.e. $\mathcal{N}_{I} \leq(C / \delta)^{1 / 2} N \eta$. Choosing $K$ sufficiently large, we obtain (5.4) from (C.5). This proves Lemma 5.3.

Lemma C.1 Let the components of the vector $\mathbf{b} \in \mathbb{R}^{N-1}$ be real i.i.d. variables with a common distribution $\mathrm{d} \nu$ that satisfies a Gaussian decay condition (2.16) for some positive $\delta_{0}>0$. Let $\xi_{\alpha}=\left|\mathbf{b} \cdot \mathbf{v}_{\alpha}\right|^{2}$, where $\left\{\mathbf{v}_{\alpha}\right\}_{\alpha \in \mathcal{I}}$ is an orthonormal set in $\mathbb{R}^{N-1}$. Then for $\delta \leq 1 / 2$ there is a constant $c>0$ such that

$$
\mathbb{P}\left\{\sum_{\alpha \in \mathcal{I}} \xi_{\alpha} \leq \delta m\right\} \leq e^{-c \sqrt{m}}
$$

holds for any $\mathcal{I}$, where $m=|\mathcal{I}|$ is the cardinality of the index set $\mathcal{I}$. 
Proof of Lemma C.1. We will need the following result of Hanson and Wright [20], extended to nonsymmetric variables by Wright [30]. We remark that this statement can also be extended to complex random variables (Proposition $4.5[10]$ ).

Proposition C.2 [20,30] Let $b_{j}, j=1,2, \ldots N$ be a sequence of real i.i.d. random variables with distribution $\mathrm{d} \nu$ satisfying the Gaussian decay (2.16) for some $\delta_{0}>0$. Let $a_{j k}, j, k=1,2, \ldots N$ be arbitrary real numbers and let $\mathcal{A}$ be the $N \times N$ matrix with entries $\mathcal{A}_{j k}:=\left|a_{j k}\right|$. Define

$$
X:=\sum_{j, k=1}^{N} a_{j k}\left[b_{j} b_{k}-\mathbb{E} b_{j} b_{k}\right]
$$

Then there exists a constant $c>0$, depending only on $\delta_{0}, D$ from (2.16), such that for any $\delta>0$

$$
\mathbb{P}(|X| \geq \delta) \leq 4 \exp \left(-c \min \left\{\delta / A, \delta^{2} / A^{2}\right\}\right),
$$

where $A:=\left(\operatorname{Tr} \mathcal{A} \mathcal{A}^{t}\right)^{1 / 2}=\left[\sum_{j, k}\left|a_{j k}\right|^{2}\right]^{1 / 2}$.

We will apply this result for

$$
X=\sum_{i, j=1}^{N} a_{i j}\left[b_{i} b_{j}-\mathbb{E} b_{i} b_{j}\right], \quad \text { with } \quad a_{i j}:=\sum_{\alpha \in \mathcal{I}} v_{\alpha}(i) v_{\alpha}(j) .
$$

Notice that $\sum_{\alpha \in \mathcal{I}} \xi_{\alpha}=X+|\mathcal{I}|=X+m$ since $\mathbb{E} \xi_{\alpha}=1$. By $\delta \leq 1 / 2$ we therefore obtain

$$
\mathbb{P}\left\{\sum_{\alpha \in \mathcal{I}} \xi_{\alpha} \leq \delta m\right\} \leq \mathbb{P}\left\{|X| \geq \frac{m}{2}\right\}
$$

Since

$$
A^{2}:=\sum_{i, j=1}^{N}\left|a_{i j}\right|^{2}=\sum_{\alpha, \beta \in \mathcal{I}} \sum_{i, j=1}^{N} v_{\alpha}(i) v_{\alpha}(j) v_{\beta}(i) v_{\beta}(j)=m,
$$

by Proposition C.2, we obtain

$$
\mathbb{P}\left\{\sum_{\alpha \in \mathcal{I}} \xi_{\alpha} \leq \delta m\right\} \leq \mathbb{P}\left\{|X| \geq \frac{m}{2}\right\} \leq 4 \exp \left(-c \min \left\{\frac{m}{2 A}, \frac{m^{2}}{4 A^{2}}\right\}\right) \leq e^{-c \sqrt{m}} .
$$

for some $c>0$.

Acknowledgement: We thank Jun Yin for several helpful comments and pointing out some errors in the preliminary versions of this paper. We are also grateful to the referees for their suggestions to improve the presentation. 


\section{References}

[1] Bakry, D., Émery, M.: Diffusions hypercontractives. in: Séminaire de probabilités, XIX, 1983/84, 1123 Lecture Notes in Mathematics, Springer, Berlin, 1985, 177-206.

[2] Ben Arous, G., Péché, S.: Universality of local eigenvalue statistics for some sample covariance matrices. Comm. Pure Appl. Math. LVIII. (2005), 1-42.

[3] Bleher, P., Its, A.: Semiclassical asymptotics of orthogonal polynomials, Riemann-Hilbert problem, and universality in the matrix model. Ann. of Math. 150 (1999): 185-266.

[4] Bobkov, S. G., Götze, F.: Exponential integrability and transportation cost related to logarithmic Sobolev inequalities. J. Funct. Anal. 163 (1999), no. 1, 1-28.

[5] Brézin, E., Hikami, S.: Correlations of nearby levels induced by a random potential. Nucl. Phys. B 479 (1996), 697-706, and Spectral form factor in a random matrix theory. Phys. Rev. E 55 (1997), $4067-4083$.

[6] Deift, P., Kriecherbauer, T., McLaughlin, K.T-R, Venakides, S., Zhou, X.: Uniform asymptotics for polynomials orthogonal with respect to varying exponential weights and applications to universality questions in random matrix theory. Comm. Pure Appl. Math. 52 (1999):1335-1425.

[7] Deift, P., Kriecherbauer, T., McLaughlin, K.T-R, Venakides, S., Zhou, X.: Strong asymptotics of orthogonal polynomials with respect to exponential weights. Comm. Pure Appl. Math. 52 (1999): $1491-1552$.

[8] Dyson, F.J.: A Brownian-motion model for the eigenvalues of a random matrix. J. Math. Phys. 3, 1191-1198 (1962).

[9] Erdős, L., Schlein, B., Yau, H.-T.: Semicircle law on short scales and delocalization of eigenvectors for Wigner random matrices. Ann. Probab. 37, No. 3, 815-852 (2009)

[10] Erdős, L., Schlein, B., Yau, H.-T.: Wegner estimate and level repulsion for Wigner random matrices. Int. Math. Res. Notices. 2010, No. 3, 436-479 (2010)

[11] Erdős, L., Ramirez, J., Schlein, B., Yau, H.-T.: Universality of sine-kernel for Wigner matrices with a small Gaussian perturbation. Electr. J. Prob. 15, Paper 18, 526-604 (2010)

[12] Erdős, L., Péché, S., Ramírez, J., Schlein, B. and Yau, H.-T.: Bulk universality for Wigner matrices. Commun. Pure Applied Math. 63, 895-925, (2010)

[13] Erdős, L., Ramírez, J., Schlein, B., Tao, T., Vu, V. and Yau, H.-T.: Bulk universality for Wigner hermitian matrices with subexponential decay. To appear in Math. Res. Letters. Preprint arXiv:0906.4400

[14] Erdős, L., Schlein, B., Yau, H.-T., Yin, J.: The local relaxation flow approach to universality of the local statistics for random matrices. Preprint arXiv:0911.3687

[15] Erdős, L., Yau, H.-T., Yin, J.: Bulk universality for generalized Wigner matrices. Preprint arXiv:1001.3453 
[16] Erdős, L., Yau, H.-T., Yin, J.: Universality for generalized Wigner matrices with Bernoulli distribution. Preprint arXiv:1003.3813

[17] Erdős, L., Yau, H.-T., Yin, J.: Rigidity of Eigenvalues of Generalized Wigner Matrices. Preprint arXiv:1007.4652

[18] Guionnet, A.: Large random matrices: Lectures on Macroscopic Asymptotics. École d'Eté de Probabilités de Saint-Flour XXXVI-2006. Springer.

[19] Gustavsson, J.: Gaussian fluctuations of eigenvalues in the GUE, Ann. Inst. H. Poincaré, Probab. Statist. 41 (2005), no.2, 151-178

[20] Hanson, D.L., Wright, F.T.: A bound on tail probabilities for quadratic forms in independent random variables. The Annals of Math. Stat. 42 (1971), no.3, 1079-1083.

[21] Johansson, K.: Universality of the local spacing distribution in certain ensembles of Hermitian Wigner matrices. Comm. Math. Phys. 215 (2001), no.3. 683-705.

[22] Ledoux, M.: The concentration of measure phenomenon. Mathematical Surveys and Monographs, 89 American Mathematical Society, Providence, RI, 2001.

[23] Lu, S.-L. and Yau, H.-T.: Spectral gap and logarithmic Sobolev inequality for Kawasaki and Glauber dynamics, Comm. Math. Phys. 156, 399-433, 1993.

[24] Mehta, M.L.: Random Matrices. Academic Press, New York, 1991.

[25] Pastur, L., Shcherbina M.: Bulk universality and related properties of Hermitian matrix models. J. Stat. Phys. 130 (2008), no.2., 205-250.

[26] Sinai, Y. and Soshnikov, A.: A refinement of Wigner's semicircle law in a neighborhood of the spectrum edge. Functional Anal. and Appl. 32 (1998), no. 2, 114-131.

[27] Soshnikov, A.: Universality at the edge of the spectrum in Wigner random matrices. Comm. Math. Phys. 207 (1999), no.3. 697-733.

[28] Tao, T. and Vu, V.: Random matrices: Universality of the local eigenvalue statistics. Preprint arXiv:0906.0510.

[29] Vu, V.: Spectral norm of random matrices. Combinatorica, 27 (6) (2007), 721-736.

[30] Wright, F.T.: A bound on tail probabilities for quadratic forms in independent random variables whose distributions are not necessarily symmetric. Ann. Probab. 1 No. 6. (1973), 1068-1070.

[31] Yau, H. T.: Relative entropy and the hydrodynamics of Ginzburg-Landau models, Lett. Math. Phys. 22 (1991) 63-80. 